\title{
Is it possible to obtain cosmic accelerated expansion through energy transfer between different energy densities?
}

\author{
Recai Erdem \\ Department of Physics, İzmir Institute of Technology, Gülbahçe, Urla 35430, İzmir, Turkey
}

\section{A R T I C L E I N F O}

\section{Article history:}

Received 28 June 2016

Received in revised form 4 December 2016

Accepted 5 December 2016

\section{Keywords:}

Accelerated cosmic expansion

Dark energy

\begin{abstract}
A B S T R A C T
The equation of state of an energy density may be significantly modified by coupling it to another energy density. In the light of this observation we check the possibility of producing cosmic accelerated expansion in this way. In particular we consider the case where matter is converted to radiation (or vice versa by particle physics processes). We find that cosmic accelerated expansion can be obtained in this way only if an intermediate state with negative equation of state forms during the conversion.
\end{abstract}

(C) 2016 Elsevier B.V. All rights reserved.

\section{Introduction}

Observations show that the universe is undergoing accelerated expansion at present, and many theoretical arguments and observational evidence suggest that the universe must have undergone an accelerated expansion period at the early times as well [1,2]. Although the standard explanations for these accelerated expansions are cosmological constant at present era and inflationary models at early times there are many alternative ways; for example, quintessence, $f(R)$ models [2], and gravitational particle production $[3,4]$. However all these models have some problems. There is a problem associated with cosmological constant called the cosmological constant problem, and it seems that the best way may be the use of some symmetry to make it cancel and seek another method for late time cosmic acceleration [5]. Inflationary models usually employ at least one new postulated scalar, and need special initial conditions, a similar situation (although less severe) is true for quintessence models. $f(R)$ type modified gravity models use an extension of general relativity, in gravitational particle production the energy density of the universe is an open thermodynamical system that is assumed to acquire energy from gravitational field while the question of if the universe is a closed system in this case is not clear enough. Therefore it is useful to seek the possibility of additional alternative ways for accelerated expansion. In particular it would be desirable to have a model where the accelerated expansion is achieved with a minimal extension of the standard models of particle physics and cosmology. In this paper, in the light of the fact that coupling an energy density to another one modifies its equation of state [6] we seek if an energy density transfer due to elementary particle processes may have the potential of providing a source for cosmic accelerated expansion. Although the analysis in

E-mail address: recaierdem@iyte.edu.tr. this paper, in principle, is applicable to all types of particle physics processes, we specify it to the case of conversion of heavy particles to light particles i.e. to the conversion of matter to radiation. In fact there must be an era of the creation of matter and radiation not only because the ordinary matter and radiation must be produced anyway but also to have a well defined model that may serve at all eras of the universe [7]. Moreover in the standard lore of cosmology the ordinary matter and radiation are assumed to be produced by the decays or the collisions of some other particles such as Higgs particle, curvaton etc. at early times [8]. Particle physics processes ranging from high energies to atomic physics have an important role at present as well. Therefore the possibility of using just matter and radiation (as in this paper) interacting through the particle physics processes for cosmic acceleration with minimal need for exotic matter is interesting. The results of the following analysis shows that obtaining cosmic acceleration through conversion of matter to radiation (or vice versa) seems impossible except through formation of an intermediate state with negative equation of state (e.g. a QCD-like condensate formed by intermediate particles produced in the particle physics processes).

In this study we consider the Robertson-Walker metric

$d s^{2}=-d t^{2}+a^{2}(t)\left[\frac{d r^{2}}{1-k r^{2}}+r^{2}\left(d \theta^{2}+\sin ^{2} \theta d \phi^{2}\right)\right]$

and for simplicity we take $k=0$ which is in agreement with observations [9]. For the illustration of the method we consider a simple case; a universe that consists of matter and radiation. We assume that, at some time $t_{1}$, the energy density of either of matter or radiation starts to be transferred to the other through some particle physics processes such as those given in Fig. 1. There may be two cases:

(i) The conversion of each matter particle to radiation may be instantaneous e.g. as in the three particle (decay) process in Fig. 1 

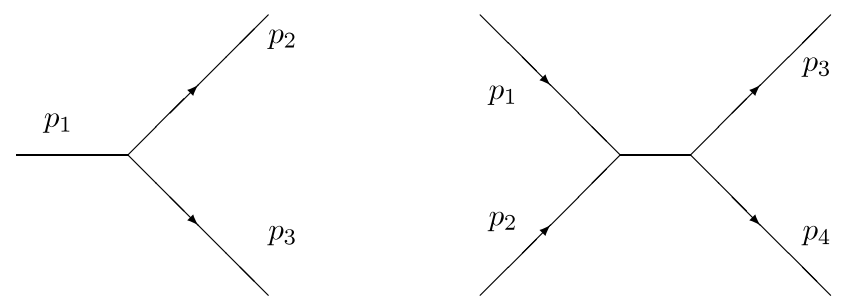

Fig. 1. The diagram on the left-hand side shows the decay of a particle with momentum $p_{1}$ into two particles with momenta $p_{2}$ and $p_{3}$ e.g. the decay of a nonrelativistic particle to two relativistic particles while the diagram on the right-hand side shows the (inelastic) collision of two particles with momenta $p_{1}$ and $p_{2}$ into two other particles with momenta $p_{3}$ and $p_{4}$ e.g. the collision of two non-relativistic particles into two relativistic particles through formation of an intermediate state.

or the four particle process in the figure where the internal line is deleted (i.e. a four particle contact interaction) or the four particle process in the figure where the process takes place in $t$ or $\mathrm{u}$ channels. This option is considered in the next section.

(ii) There may be an on-shell intermediate particle in the process e.g. the four point process in the figure where the process takes place in s channel i.e. a resonance forms in the intermediate state in the conversion of matter to radiation. This option is considered in the section after the next section. We consider the two cases mentioned above separately. In both cases above we take the matter to be extremely non-relativistic and approximate its cosmological evolution by that of dust while we assume that the matter particles have yet non-vanishing small velocities enabling them to participate in particle processes such as scattering.

\section{The case of instantaneous interactions}

In this case the interaction between individual particles is instantaneous as in the three particle process or the four particle process in $t$ or $u$ channels in Fig. 1. In this case the total energy density of the universe may be written as

$$
\begin{aligned}
& \rho(t)=\rho_{m}(t)+\rho_{r}(t) \\
& \rho_{m}(t)=\frac{C_{m}(t)}{[a(t)]^{3}}, \quad \rho_{r}(t)=\frac{C_{r}(t)}{[a(t)]^{4}}
\end{aligned}
$$

where $a(t)$ is the scale factor (of Robertson-Walker metric) at time $t$, and (see Appendix A)

$$
\begin{aligned}
& C_{m}(t)=\rho_{m 0}-\int_{t_{1}}^{t}\left(\frac{d \tilde{\rho}\left(t^{\prime}\right)}{d t^{\prime}}\right) a^{3}\left(t^{\prime}\right) d t^{\prime} \\
& C_{r}(t)=\rho_{r 0}+\int_{t_{1}}^{t}\left(\frac{d \tilde{\rho}\left(t^{\prime}\right)}{d t^{\prime}}\right) a^{4}\left(t^{\prime}\right) d t^{\prime}
\end{aligned}
$$

where $\frac{\tilde{\rho}(t)}{d t}$ is the rate of the energy density transfer from matter to radiation (in the comoving frame). In this case the effect of interaction is absorbed into the change in evolution of the energy densities of matter and radiation through time dependence of $C_{m}(t)$ and $C_{r}(t)$.

We may easily see from (3), (4), (5) that $\rho_{m}(t), \rho_{r}(t)$ and $\rho(t)=$ $\rho_{m}(t)+\rho_{r}(t)$ satisfy the following equations (see Appendix A)

$$
\begin{aligned}
& \dot{\rho}_{m}(t)+3 H \rho_{m}(t)=\frac{\dot{C}_{m}(t)}{[a(t)]^{3}}=-\frac{d \tilde{\rho}(t)}{d t} \\
& \dot{\rho}_{r}(t)+4 H \rho_{r}(t)=\frac{\dot{C}_{r}(t)}{[a(t)]^{4}}=\frac{d \tilde{\rho}(t)}{d t} \\
& \frac{\dot{C}_{m}(t)}{a^{3}}+\frac{\dot{C}_{r}(t)}{a^{4}}=0 \\
& \dot{\rho}(t)+3 H\left[1+\frac{1}{3} \frac{\rho_{r}(t)}{\rho(t)}\right] \rho(t)=0 .
\end{aligned}
$$

In other words the interaction that converts matter to radiation does not induce an additional energy density or pressure, so it does not affect the overall evolution rate of the universe while it affects the evolution of each component, matter and radiation separately. In fact this conclusion holds in a more general context. Consider two energy densities, $\rho_{1}$ and $\rho_{2}$ with equations of states $\omega_{1}$ and $\omega_{2}$, respectively in the absence of any interaction between $\rho_{1}$ and $\rho_{2}$; then allow an energy density transfer $Q$ between $\rho_{1}$ and $\rho_{2}$. The situation is similar to Eqs. (6), (7), and (9) where $\frac{d \tilde{\rho}}{d t}$ is replaced by $Q$, namely,

$$
\begin{aligned}
& \dot{\rho}_{1}(t)+3 H(t)\left(1+\omega_{1}(t)\right) \rho_{1}(t)=Q(t) \\
& \dot{\rho}_{2}(t)+3 H(t)\left(1+\omega_{2}(t)\right) \rho_{2}(t)=-Q(t) \\
& \dot{\rho}(t)+3 H(1+\omega(t)) \rho(t)=0 \\
& \text { where } \rho(t)=\rho_{1}(t)+\rho_{2}(t), \quad \omega(t)=\frac{\omega_{1} \rho_{1}(t)+\omega_{2} \rho_{2}(t)}{\rho_{1}(t)+\rho_{2}(t)} .
\end{aligned}
$$

In fact the form of the Eqs. (10) and (11) are familiar from the models of dark matter-dark energy interactions [10] where dark matter and dark energy are replaced by (dark) matter and radiation. It is evident from the form of (13) that $\omega$ is positive for all times if both $\omega_{1}$ and $\omega_{2}$ are positive at some initial time and $\omega$ is negative for all times if both $\omega_{1}$ and $\omega_{2}$ are negative at some initial time (provided that $\rho_{1(2)}$ is always positive). In other words $\omega_{1}$ and $\omega_{2}$ should have different signs if one wants to construct a model where the dark energy is subdominant initially and at later time becomes dominant or vice versa (and introduction of $Q$ does not have any effect on the time of transition between $\omega>0$ and $\omega<0$ eras). Therefore one cannot induce a dark energy effect through conversion of matter to radiation (or vice versa) in this way.

For example, assume that the conversion rate of matter and radiation is such that $-\frac{d \tilde{\rho}(t)}{d t}=3 H \rho_{m}$. Then (6), (7) become

$$
\begin{aligned}
& \dot{\rho}_{m}(t)+3 H \rho_{m}(t)=3 H \rho_{m}(t) \Rightarrow \rho_{m}(t)=\text { constant }=\rho_{0} \\
& \dot{\rho}_{r}(t)+4 H \rho_{r}(t)=-3 H \rho_{m}(t)=-3 H \rho_{0} .
\end{aligned}
$$

Hence

$\frac{4}{3} \rho_{r}+\rho_{0}=A e^{-3 \int H d t}$

where $A$ is some integration constant. In other words $\rho_{m}$ in this example behaves as cosmological constant (although in the absence of an energy density transfer between matter and radiation $\rho_{m}$ scales as $\frac{1}{a^{3}}$ ), and $\rho_{r}$ behaves as an exotic fluid with the energy density given in (16). Therefore introduction of an energy transfer between matter and radiation may considerably change their cosmic evolution and an otherwise standard energy density may seem exotic due to the coupling of that energy density to another energy density. However if one adds the differential equations in (14) and (15) one finds that the equation is the same as (9) i.e. it is the same as the evolution of an energy density composed of matter and radiation that do not interact with each other. In other words, although the evolution of each energy density component in total energy density may drastically change due to introduction of an energy transfer between different components of the total energy density, the overall evolution of the total energy density remains the same after the introduction of the energy transfer.

\section{The case of a resonance as the intermediate state in the conversion of matter to radiation}

\subsection{General discussion}

This is the case where the internal line in the four particle process in Fig. 1 is an on-shell s-channel intermediate particle (i.e. 
resonance). In this case the total energy density of the universe may be written as

$\rho(t)=\rho_{m}(t)+\rho_{r}(t)+\rho_{R}(t)$

where $\rho_{m}(t)$ and $\rho_{r}(t)$ are the energy densities of matter and radiation given by (2) where $C_{m}(t)$ and $C_{r}(t)$ are replaced by

$$
\begin{aligned}
& C_{m}(t)=\rho_{m 0}-\int_{t_{1}}^{t}\left(\frac{d \tilde{\rho}_{m}\left(t^{\prime}\right)}{d t^{\prime}}\right) a^{3}\left(t^{\prime}\right) d t^{\prime} \\
& C_{r}(t)=\rho_{r 0}+\int_{t_{1}}^{t}\left(\frac{d \tilde{\rho}_{r}\left(t^{\prime}\right)}{d t^{\prime}}\right) a^{4}\left(t^{\prime}\right) d t^{\prime} .
\end{aligned}
$$

Because the form of the energy density of the intermediate state i.e. the resonance $\rho_{R}(t)$ is not specified it has a general form given by

$\rho_{R}(t)=e^{-\Gamma\left(t-t_{1}\right)} C_{R}(t) \exp \left[-3 \int_{a\left(t_{1}\right)}^{a(t)} \frac{\left(1+\omega_{R}\left(a^{\prime}\right)\right) d a^{\prime}}{a^{\prime}}\right]$.

Note that $e^{-\Gamma\left(t-t_{1}\right)}$ term would not be present in (20) if we had taken the resonance as stable, where $\Gamma$ is the width of the resonance. $C_{R}$ in (20) may be obtained in the same way as done in Appendix $A$ as

$$
\begin{aligned}
C_{R}(t)= & \int_{t_{1}}^{t} d t^{\prime}\left[\left(\frac{d \tilde{\rho}_{m}\left(t^{\prime}\right)}{d t^{\prime}}\right) e^{\Gamma\left(t^{\prime}-t_{1}\right)}\right] \\
& \times \exp \left[3 \int_{a\left(t_{i}\right)}^{a\left(t^{\prime}\right)} \frac{\left(1+\omega_{R}(\tilde{a})\right) d \tilde{a}}{\tilde{a}}\right]
\end{aligned}
$$

where we have used $\frac{d \tilde{\rho}_{R}\left(t^{\prime}\right)}{d t^{\prime}}=\frac{d \tilde{\rho}_{m}\left(t^{\prime}\right)}{d t^{\prime}}$ since the amount of energy transferred from the matter is equal to the one acquired by the resonance. $\rho_{R}$ satisfies

$$
\begin{aligned}
& \dot{\rho}_{R}+3 H\left(1+\omega_{R}\right) \rho_{R} \\
& =e^{-\Gamma\left(t-t_{1}\right)} \dot{C}_{R}(t) \exp \left[-3 \int_{a\left(t_{i}\right)}^{a(t)} \frac{\left(1+\omega\left(a^{\prime}\right)\right)}{a^{\prime}}\right] \\
& -\Gamma e^{-\Gamma\left(t-t_{1}\right)} C_{R}(t) \exp \left[-3 \int_{a\left(t_{i}\right)}^{a(t)} \frac{\left(1+\omega\left(a^{\prime}\right)\right)}{a^{\prime}}\right] \\
& =\frac{d \tilde{\rho}_{R}(t)}{d t}-\Gamma \rho_{R}
\end{aligned}
$$

which is equivalent to

$\dot{\rho}_{R}+3 H\left(1+\omega_{R}+\Delta \omega_{R}\right) \rho_{R}=0$

where

$\Delta \omega_{R}=\frac{1}{3 H}\left(-\frac{\frac{d \tilde{\rho}_{R}(t)}{d t}}{\rho_{R}}+\Gamma\right)$.

Eq. (24) tells us that the effect induced by the formation of the resonance will act like dark energy if the ratio of the rate of conversion of the energy (of the matter to the resonance) to the energy density of the resonance is higher than the decay rate of the resonance. Note that this effect is independent of the background effect produced by the resonance i.e. $\Delta \omega_{R}$ is independent of $\omega_{R}$ in general.

Note that we had taken $\frac{d \tilde{\rho}_{m}}{d t}=\frac{d \tilde{\rho}_{r}}{d t}$ in the previous case because the conversion of each matter particle to radiation was instantaneous while here $\frac{d \tilde{\rho}_{m}}{d t}=\frac{d \tilde{\rho}_{\mathrm{r}}}{d t}$ since there is a resonance as the intermediate state in this case hence the rate of the conversion of matter to the resonance, $\frac{d \tilde{\rho}_{m}}{d t}$ may not be equal to the rate of the conversion of the energy density of the resonance to radiation $\frac{d \tilde{\rho}_{r}}{d t}$ at a specific time. Instead we should have

$$
\begin{aligned}
& -\frac{d \tilde{\rho}_{m}}{d t}+\frac{d \tilde{\rho}_{r}}{d t}+\frac{d \tilde{\rho}_{m}}{d t}-\Gamma \rho_{R} \\
& =\frac{\dot{C}_{m}(t)}{a^{3}}+\frac{\dot{C}_{r}(t)}{a^{4}}+\left[\frac{d\left(e^{-\Gamma\left(t-t_{1}\right)} C_{R}(t)\right)}{d t}\right] \\
& \quad \times \exp \left[-3 \int_{a\left(t_{1}\right)}^{a(t)} \frac{\left(1+\omega\left(a^{\prime}\right)\right)}{a^{\prime}}\right]=0 \\
& \text { i.e. } \quad \frac{d \tilde{\rho}_{r}}{d t}=\Gamma \rho_{R} .
\end{aligned}
$$

In other words the net change in the total energy due to the energy transfers between matter, radiation, and the resonance state should be zero at each time $t$ since we assume that system is a closed system with no energy transfer from outside, and the source of radiation is the decay of the resonance. Eq. (25) tells us that there is no direct effect of the interaction (of the conversion of energy densities) on the total energy density, instead the effect of conversion is indirect and through the evolutions of $\dot{C}_{m}(t), \dot{C}_{r}(t), \dot{C}_{R}(t)$ as in the preceding subsection. In other words by taking different sets of $\dot{C}_{m}(t), \dot{C}_{r}(t), \dot{C}_{R}(t)$ one may mimic different effective energy densities in this case as well while the evolution of the total energy density does not change after introduction of conversion between energy densities of matter and radiation. The only way to induce a dark energy effect in this way is to assume that the resonance state has $\omega<0$ (e.g. the resonance particles form a QCD-like condensate). In fact this may be anticipated from (13); one may introduce a third energy density $\rho_{3}$ with $\omega_{3}<0$ (or a variable $\omega_{3}$ that becomes $\omega_{3}<0$ at some time during its evolution) so that the equation state of the universe, $\omega$ may change sign during the evolution of the energy densities while it is impossible in the case of a universe that only consist of matter and radiation with $\omega_{1}>0, \omega_{2}>0, \rho_{1}>0, \rho_{2}>0$. In this subsection we want to focus on the effect of the resonance and possibility of taking it as an energy density inducing an accelerated cosmic expansion.

One may describe the general evolution of this system as follows: The evolutions of $C_{m}$ and $C_{R}$ are given by (18) and (21), respectively. The evolution of $C_{r}$ is given by (19) where (26) is employed. For simplicity we take $\rho_{r 0}=0$ i.e.

$C_{r}(t)=\Gamma \int_{t_{1}}^{t} \rho_{R}\left(t^{\prime}\right) a^{4}\left(t^{\prime}\right) d t^{\prime}$.

Then at $t=t_{1} C_{m}$ (so, $\rho_{m}$ ) is maximum, and $C_{R}$ (so, $\rho_{R}$ ) and $C_{r}$ (so, $\left.\rho_{r}\right)$ are zero. By time $C_{m}$ decreases till $t=t_{m f}$ when $C_{m}$ becomes small or $C_{m} \simeq 0$ and $C_{R}$ is maximum and $C_{r}$ has a small finite value. At $t>t_{m f} C_{m}$ gets even closer to zero (if it is not zero yet), $e^{-\Gamma\left(t-t_{1}\right)} C_{R}(t)$ gets smaller by time and becomes zero as $t \rightarrow \infty$ while $C_{r}$ gets its maximum at $t \rightarrow \infty$. In other words the evolution of the system may be summarized as: Initially the universe is matter dominated then it becomes resonance dominated and eventually the universe becomes radiation dominated. In the next subsection we will describe the resonance state by resonance particles produced by the particle physics process given in the second diagram in Fig. 1 for relatively simple cases.

\subsection{A particle physics description}

We may adopt the derivation for the formula of energy density of matter in Appendix A (i.e. Eq. (18)) to find an expression for number densities since both have the same dependence on scale factor i.e. both vary as $\propto a^{-3}$. To find the corresponding expression for number densities we replace $\frac{d \tilde{\rho}(t)}{d t}$ by $\frac{d \tilde{n}(t)}{d t}$ and $\rho_{m}(t)$ by $n(t)$. Then we may employ the method we had used for energy densities 
in Appendix A to write a similar expression for number densities of stable particles

$$
\begin{aligned}
n(t)= & \frac{C^{(n)}(t)}{[a(t)]^{3}}, \quad C^{(n)}=n_{1}-\int_{0}^{t-t_{1}}\left(\frac{d \tilde{n}(x)}{d x}\right)_{x=(t-u)} \\
& \times a^{3}(t-u) \theta(u) d u
\end{aligned}
$$

where we have put the superscript ${ }^{(n)}$ to the coefficient $C$ to distinguish it from the corresponding symbol for energy densities, $n_{1}$ stands for the initial value of $n(t)$ at the initial time $t=t_{1}$ of the energy transfer, and $\frac{d \tilde{n}(t)}{d t}$ stands for the number density transferred per time in per comoving volume i.e. the rate of the number density transfer in the comoving frame, and $\theta(u)$ is the Heaviside function (i.e. unit step function) given by $\theta=0$ if $u<0$ and $\theta=1$ if $u>0$. Eq. (28) (after using a derivation similar to the one for matter in Appendix A) implies that

$\frac{d \tilde{n}(t)}{d t}=-\frac{\dot{C}^{(n)}(t)}{[a(t)]^{3}}$.

The rate of the conversion of the matter to resonance particles by collisions of two beams of matter particles, each with number densities $\frac{n_{m}}{2}$ may be expressed as

$$
-\frac{d \tilde{n}_{m}(t)}{d t}=\frac{\dot{C}_{m}^{(n)}(t)}{[a(t)]^{3}}=-\beta^{\prime} n_{m}^{2}(t) \sigma(t) v(t)
$$

where $\frac{1}{4}>\beta^{\prime}>0$ is some constant designating the effective penetration depth of a beam of $\frac{n_{m}}{2}$ to a target of $\frac{n_{m}}{2}$ particles in unit comoving volume, $\sigma$ is the cross section of the four-point Feynman diagram in Fig. 1, $v$ is the average velocity of the (non-relativistic) particles relative to the comoving frame. For later reference we need dependence of cross section on scale factor. The differential cross section $d \sigma$ of two initial particles of 4-momenta $p_{1}, p_{2}$ and masses $m_{1}, m_{2}$ going to a final state of two particles of 4-momenta $p_{1}^{\prime}$ and $p_{2}^{\prime}$ in local Minkowski space is given by [11]

$d \sigma=\frac{(2 \pi)^{4} \delta^{4}\left(p_{1}+p_{2}-p_{1}^{\prime}-p_{2}^{\prime}\right)}{4 E_{1} E_{2} v} \frac{d^{3} \mathbf{p}_{1}^{\prime}}{(2 \pi)^{3} 2 E_{1}^{\prime}} \frac{d^{3} \mathbf{p}_{2}^{\prime}}{(2 \pi)^{3} 2 E_{2}^{\prime}}\left|M_{f i}\right|^{2}$

where bold face quantities denote 3-dimensional quantities, $E$ denotes energies, the subindices 1 and 2 denote the 1st and the 2nd particles, unprimed letters correspond to initial state particles and the primed ones correspond to final state particles. $E_{j}$ denotes energy of the $\mathrm{j}$ 'th particle, and $M_{f i}$ is the transition amplitude from initial state to the final state. The dependence of the cross section on scale factor is through its dependence on momenta in (31). After integrating over final momenta the apparent dependence of the cross section on $\mathbf{p}_{1(2)}^{\prime}$ disappears. Therefore the dependence of the cross section on scale factor is through $E_{1(2)}, E_{1(2)}^{\prime}$, and $\left|M\left(p_{1}, p_{2} ; k_{i}\right)\right|^{2}$. In this case $E_{1(2)}$ are extremely non-relativistic, so they may essentially be taken constant. $E_{1(2)}^{\prime}$ are extremely relativistic, so may be taken to vary proportional to $\frac{1}{a}$. $M_{i f}$ corresponding to the four-point process in figure in the perturbative limit for an on-shell intermediate state (in s-channel) is given by $[11,12]$

$M_{f i}=\left(-i g_{1}\right)\left(-i g_{2}\right)\left(\frac{1}{s-m_{R}^{2}+i \sqrt{s} \Gamma_{R}}\right)$

where $g_{1}, g_{2}$ are coupling constants of the interactions in the vertices, $s=\left(p_{1}+p_{2}\right)^{2}$, and $m_{R}, \Gamma_{R}$ are the mass, the decay width of the resonance, respectively. Because the initial state consist of extremely non-relativistic particles one may write

$\left(p_{1}+p_{2}\right)^{2} \simeq\left(E_{1}+E_{2}\right)^{2} \simeq\left(2 m+m v^{2}\right)^{2}, \quad m_{R} \simeq 2 m$.

Therefore for the values of $s$ near the pole of the resonance

$s-m_{R}^{2}+i \sqrt{s} \Gamma_{R}(s) \simeq m_{R}^{2} v^{2}+i m_{R} \Gamma_{R}(s)$
$\Gamma_{R}$ is also a function of $s$ in general, as emphasized in (34), so the form of dependence of $\Gamma_{R}$ on $s$ must be also known to determine the dependence of cross section on scale factor. In the case of perturbative field theory $\Gamma_{R}$ is identified as the imaginary part of self interaction and can be calculated in principle. In the case of a perturbative quantum field theory consisting of only scalars with 3-point interactions the leading order contribution is found to be proportional to $\sqrt{s}[13]$. When the theory gets non-perturbative higher order terms that depend on higher powers $a$ are expected to become dominant. In fact in this study we consider extremely narrow resonances (i.e. $\Gamma \ll m_{R}$ ) since the lifetime of the resonance must be long enough to be cosmologically relevant, so the last term in (34) may be neglected. Therefore

$M_{f i} \propto a^{p}(t)$

where $p=2$ in the case of an extremely narrow resonance. Hence we find that

$d \sigma \propto a^{r}(t)$ where in the case of an extremely

narrow resonance $r=7$.

The same result may be obtained from the general expression $M_{f i}$ for the values of the total energy close to the resonance pole $[9,14]$

$M_{f i}=-\Gamma_{1} \Gamma_{2}\left(\frac{1}{s-m_{R}^{2}+i \sqrt{s} \Gamma_{R}}\right)$

where $\Gamma_{1(2)}$ are the partial widths of the resonance associated with the first and second vertex in Fig. 1. While (37) is more general in the sense that it is not restricted to perturbative interactions, it may not be evident that $\Gamma_{1(2)}$ do not vary with scale factor.

The energy density of dust particles is proportional to its number density. We assume the matter particles are extremely nonrelativistic so their energy density may be approximated by that of dust. Therefore (30) may be rewritten as

$$
\begin{aligned}
\frac{d \tilde{\rho}_{m}(t)}{d t} & =E \frac{d \tilde{n}_{m}(t)}{d t}=-\frac{\dot{C}_{m}(t)}{[a(t)]^{3}} \\
& =\beta \rho_{m}^{2}(t) a^{r-1}(t), \beta=\frac{\beta^{\prime} \sigma\left(t_{1}\right) v\left(t_{1}\right)}{E a\left(t_{1}\right)}
\end{aligned}
$$

where $E \simeq m$ is the average energy of the matter particles. In (38) we have used Eq. (35) i.e. $\sigma(t)=\sigma\left(t_{1}\right)\left(\frac{a(t)}{a\left(t_{1}\right)}\right)^{r}$ (where we have used the expression for cross section in local Minkowski space), and the fact that $v(t)=v\left(t_{1}\right) \frac{a\left(t_{1}\right)}{a(t)}$, and $E$ is taken to be constant since particles are taken to be extremely non-relativistic and it becomes even more non-relativistic by time due to redshift. After using $\rho_{m}(t)$ in (3), (38) may be rearranged as

$$
\begin{aligned}
\frac{\dot{C}_{m}(t)}{[a(t)]^{3}}=-\theta & \left.t-t_{1}\right) \beta\left(\frac{C_{m}(t)}{[a(t)]^{3}}\right)^{2} a^{r-1}(t) \\
\Rightarrow \quad \frac{d C_{m}}{C_{m}^{2}}= & -\theta\left(t-t_{1}\right) \beta a^{r-4}(t) d t=-\theta\left[a(t)-a\left(t_{1}\right)\right] \\
& \times \frac{\beta d a}{a^{5-r} H} .
\end{aligned}
$$

Eq. (40) may be used to determine $C_{m}(t)$. This, in turn, may be used to find $\frac{d \tilde{\rho}_{m}(t)}{d t}$ from (38), and $\rho_{R}$ from (20), and so $\Delta \omega_{R}$ from (24). This will be done below for a relatively simple, yet phenomenologically relevant case.

Next we find $C_{m}(t)$ for some choices of $H$ by using (40). For the sake of simplicity consider the cases where

$H=\xi a^{s}(t)$.

Here $\xi$ and $s$ are some constants. In fact (41) contains most of the phenomenologically relevant simple cases i.e. radiation, matter, 


$$
\begin{aligned}
C_{m} & =\frac{\xi \rho_{m 0}(4-r+s) a^{4-r+s}\left(t_{1}\right) a^{4-r+s}(t)}{-\beta \rho_{m 0} \theta\left(t-t_{1}\right)\left[a^{4-r+s}\left(t_{1}\right)-a^{4-r+s}(t)\right]+\xi(4-r+s) a^{4-r+s}\left(t_{1}\right) a^{4-r+s}(t)} \\
& =\frac{\xi \rho_{m 0}(4-r+s)}{\xi(4-r+s)+\beta \rho_{m 0} \theta\left(t-t_{1}\right)\left[a^{r-s-4}\left(t_{1}\right)-a^{r-s-4}(t)\right]}
\end{aligned}
$$

Box I.

cosmological constant dominated eras. After using (41), (40) becomes

$$
\begin{aligned}
-\frac{1}{C_{m}(t)} & =-\theta\left(t-t_{1}\right) \frac{\beta}{\xi} \int \frac{d a}{a^{5-r+s}}-\frac{1}{C_{m}\left(t_{1}\right)} \\
& =\frac{\beta \theta\left(t-t_{1}\right)}{\xi(4-r+s)}\left[\frac{1}{a^{4-r+s}(t)}-\frac{1}{a^{4-r+s}\left(t_{1}\right)}\right]-\frac{1}{\rho_{m 0}}
\end{aligned}
$$

where we have used $C_{m}\left(t_{1}\right)=\rho_{m 0}$. (42) results in (43) given in Box I. Eq. (43) implies that $s \rightarrow r-4$ limit is only well-defined for $\beta=0$ and is constant, and becomes not-well defined if $\beta \neq 0$. This suggests that at a universe where $s=r-4$ the processes in Fig. 1 cannot take place. Moreover $C_{m} \rightarrow 0$ as $a(t) \rightarrow \infty$ for $r-s-4>0$ while $C_{m}$ goes a non-zero constant value as $a(t) \rightarrow \infty$ for $r-s-4<0$. This implies that this process is not feasible in the case $r-s-4<0$ for an expanding universe.

Eq. (43) results in

$$
\begin{aligned}
& -\frac{d \tilde{\rho}_{m}(t)}{d t}=\frac{\dot{C}_{m}(t)}{a^{3}(t)} \\
& =-\frac{\theta\left(t-t_{1}\right) \rho_{m 0}^{2} \beta \xi^{2}(4-r+s)^{2} a^{r-7}(t)}{\left\{\xi(4-r+s)+\beta \rho_{m 0} \theta\left(t-t_{1}\right)\left[a^{r-s-4}\left(t_{1}\right)-a^{r-s-4}(t)\right]\right\}^{2}}
\end{aligned}
$$

$\rho_{m}(t)$

$$
=\frac{C_{m}}{a^{3}}=\frac{\xi \rho_{m 0}(4-r+s) a^{-3}(t)}{\xi(4-r+s)+\beta \rho_{m 0} \theta\left(t-t_{1}\right)\left[a^{r-s-4}\left(t_{1}\right)-a^{r-s-4}(t)\right]} .
$$

\subsubsection{Evolution of the energy densities through an example}

Now we consider an example to check if one may realize the correct evolution of the total energy density in a consistent way. To check the possibility of the resonance obtained during conversion of matter to radiation that serve as dark energy we take the simplest choice i.e. the case where the resonance particles induce a condensate that behaves as cosmological constant. We consider, first, a matter dominated universe for $t_{1}<t<t_{2}$, where $s=$ $-\frac{3}{2}$ (that may be taken as the universe just after the conversion of matter particles to the resonance state), then a cosmological constant-like resonance dominated universe where $s=0$ for $t_{2}<t<t_{3}$, finally, the radiation dominated era at the end where $s=-2$ for $t_{3}<t$. For simplicity we assume abrupt changes in the equations of states as one passes from one era to the other although in a more realistic case the changes would be smooth. However this choice is enough to give the essentials points. We take $r=7$ i.e. we take the intermediate state resonance be an extremely narrow resonance (to have a sufficiently long lifetime). In other words we consider the case

$\omega_{R}=-1, \quad r=7 \quad$ and

Initially $s=-\frac{3}{2}, \quad$ in between $s=0, \quad$ at late times $s=-2$.

We will assume that $\beta$ is constant in all eras. We give $C_{m}, \rho_{R}, C_{r}$ in these eras below by using the formulas derived above. (Please refer to Appendix B for the details of the derivations). $\rho_{m}$ and $\rho_{r}$ are evident from $C_{m}$ and $C_{r}$.
$C_{m}(t)$ at different eras are found as

$$
\text { For } t_{1}<t<t_{2} \quad C_{m}=\frac{\rho_{m 0}}{1+\frac{2 \beta \rho_{m 0}}{9 \xi_{1}}\left(a^{\frac{9}{2}}-a_{1}^{\frac{9}{2}}\right)}
$$

where $a=a(t), a_{1}=a\left(t_{1}\right)$, and (47) is directly found from (43) for $r=7, s=s_{1}=-\frac{3}{2}, \xi=\xi_{1}$.

$$
\begin{aligned}
& \text { For } t_{2}<t<t_{3} \\
& \begin{aligned}
& C_{m}(t)=\rho_{m 0}\left\{1+\rho_{m 0} \beta\left[\frac{2}{9} \xi_{1}^{-1}\left(a_{2}^{\frac{9}{2}}-a_{1}^{\frac{9}{2}}\right)\right.\right. \\
&\left.\left.+\frac{1}{3} \xi_{2}^{-1}\left(a^{3}-a_{2}^{3}\right)\right]\right\}^{-1}
\end{aligned}
\end{aligned}
$$

where $a_{2}=a\left(t_{2}\right)$, and to find $C_{m}$ in this case we have divided the integral in (42) into two parts; one for the era when $s=-\frac{3}{2}$ between $t_{1}$ and $t_{2}$, and then the era when $s=0, \xi=\xi_{2}$. between $t_{2}$ and $t$. In a similar way as in (48) we find

For $t_{3}<t \quad C_{m}(t)=\frac{\rho_{m 0}}{A^{\prime}+B^{\prime} a^{5}}$.

Here $A^{\prime}, B^{\prime}$ are some constant whose explicit forms are given in (B.21). We notice that $C_{m}$ starts from $\rho_{m 0}$ and goes to zero as $a \rightarrow$ $\infty$ as expected.

$\rho_{R}$ 's in these eras are found as

$$
\begin{aligned}
& \text { For } t_{1}<t<t_{2} \\
& \rho_{R}(t)=e^{-\Gamma\left(t-t_{1}\right)} \frac{C_{R}}{a^{3}} \simeq \frac{2}{3} \frac{\rho_{m 0}^{2} \beta}{\xi_{1}} \frac{1}{a^{3}(t)}\left[a^{\frac{3}{2}}-a_{1}^{\frac{3}{2}}\right]
\end{aligned}
$$

For $t_{2}<t<t_{3}$

$$
\begin{gathered}
\rho_{R}(t)=\left(\frac{a_{1}}{a}\right)^{3} \frac{1}{3}\left[\frac{2 \rho_{m 0}^{2} \beta}{\xi_{1}}\left\{a_{2}^{\frac{3}{2}}-a_{1}^{\frac{3}{2}}\right\}\right. \\
\left.+\frac{\rho_{m 0}^{2} \beta}{B \xi_{2}}\left\{\frac{1}{A}-\frac{1}{A+B a^{3}}\right\}\right]
\end{gathered}
$$

where have taken $\gamma=\frac{\Gamma}{H}=3$ in (B.12) as a generic case where the integration is simpler, and $A, B$ are some constants whose explicit forms are given in (B.13) and (B.14). For $t>t_{3}$

$$
\begin{aligned}
\rho_{R}(t)= & e^{-\Gamma\left(t-t_{1}\right)} C_{R}(t) \\
\simeq & \frac{4096 \xi_{3}^{4} \rho_{m 0}^{2} \beta}{3 e^{4} \Gamma^{4} \xi_{1}}\left(a^{2}-a_{1}^{2}\right)^{-4} \\
& \times\left[2\left(a_{2}^{\frac{3}{2}}-a_{1}^{\frac{3}{2}}\right)+\frac{a_{1}^{\frac{3}{2}}}{B}\left(1-\frac{1}{1+B a_{3}^{3}}\right)\right]
\end{aligned}
$$

where we have essentially employed (6), (21), (20), and (47), (48), (49). One notices that $\rho_{R}$ starts from zero at $t=0$ and keeps rising at intermediate times and goes to zero due to its decay as $a \rightarrow \infty$ as expected. In fact one may also refer to the behavior of $C_{R}$ in Appendix B to see that $C_{R}$ is zero first and goes to a constant value as $a \rightarrow \infty$. $\rho_{R}$ would behave like a cosmological constant at large scales factors if it had not decayed. 
The corresponding $C_{r}$ 's are

$$
\begin{aligned}
& \text { For } t_{1}<t<t_{2} \\
& \begin{array}{c}
C_{r}(t)=\frac{2 \Gamma \rho_{m 0}^{2} \beta}{\xi_{1}^{2}} \int_{a_{1}}^{a}\left(a^{\prime 3}-a_{1}^{\frac{3}{2}} a^{\prime \frac{3}{2}}\right) d a^{\prime} \\
=\frac{\Gamma \rho_{m 0}^{2} \beta}{2 \xi_{1}^{2}}\left[a^{4}-\frac{8 a_{1}^{\frac{3}{2}}}{5} a^{\frac{5}{2}}+\frac{3}{5} a_{1}^{4}\right]
\end{array}
\end{aligned}
$$

For $t_{2}<t<t_{3}$

$$
\begin{aligned}
C_{r}(t) & \simeq \frac{\Gamma \rho_{m 0}}{\xi_{1}}\left[\frac{\rho_{m 0} \beta}{2 \xi_{1}}\left(a_{2}^{4}-\frac{8 a_{1}^{\frac{3}{2}}}{5} a_{2}^{\frac{5}{2}}+\frac{3}{5} a_{1}^{4}\right)\right. \\
& \left.+\left(\frac{2 \rho_{m 0} \beta a_{1}^{3}\left(a_{2}^{\frac{3}{2}}-a_{1}^{\frac{3}{2}}\right)}{3 \xi_{2}}\right)\left(a-a_{2}\right)\right]
\end{aligned}
$$

For $t_{3}<t$

$$
\begin{aligned}
C_{r}(t) & \simeq \frac{\Gamma \rho_{m 0}}{\xi_{1}}\left[\frac{\rho_{m 0} \beta}{2 \xi_{1}}\left(a_{2}^{4}-\frac{8 a_{1}^{\frac{3}{2}}}{5} a_{2}^{\frac{5}{2}}+\frac{3}{5} a_{1}^{4}\right)\right. \\
& \left.+\left(\frac{2 \rho_{m 0} \beta a_{1}^{3}\left(a_{2}^{\frac{3}{2}}-a_{1}^{\frac{3}{2}}\right)}{3 \xi_{2}}+a_{1}^{\frac{9}{2}}\right)\left(a_{3}-a_{2}\right)\right] \\
& +C^{\prime \prime}\left[\frac{a_{1}^{2}-3 a_{1}^{2} a^{2}+3 a^{4}}{6\left(a^{2}-a_{1}^{2}\right)}-\frac{a_{1}^{2}-3 a_{1}^{2} a_{3}^{2}+3 a_{3}^{4}}{6\left(a_{3}^{2}-a_{1}^{2}\right)}\right]
\end{aligned}
$$

where $C^{\prime \prime}$ is a constant whose explicit form is given in (B.33), and $C_{r}$ 's are found by using (27) and (50), (51), (52). We notice that $C_{r}$ starts from zero and goes to a constant value as $a$ increases as expected.

\subsubsection{A concrete particle physics model}

In this sub-subsection, through a concrete particle physics model, we illustrate the main lines of the results of our analysis on the use of on-shell intermediate state particle condensates for cosmic accelerated expansion. In fact many scalar fields in cosmology including inflaton or quintessence fields may be considered as Bose-Einstein condensates in the light of their homogeneity and coherence [15]. This approach is elaborated and detailed in many models of dark matter [16,17], and a few models of dark energy [18-20] and inflation [21,22]. We study a specific model of dark energy similar to a model in literature [19] to see how the formulation developed in the previous parts of this subsection provides additional insight and improvement. The model we consider is simple yet sophisticated enough to be able to study main lines of this scheme in a proper way.

We consider the following Lagrangian

$$
\begin{aligned}
\mathcal{L}= & \sqrt{-g}\left\{-g^{\mu \nu}\left(\partial_{\mu} \phi^{*} \partial_{\nu} \phi+\partial_{\mu} \chi_{m 1}^{*} \partial_{\nu} \chi_{m 1}\right.\right. \\
& \left.+\frac{1}{2} \partial_{\mu} \chi_{m 2} \partial_{\nu} \chi_{m 2}+\partial_{\mu} \chi_{r 1}^{*} \partial_{\nu} \chi_{r 1}+\frac{1}{2} \partial_{\mu} \chi_{r 2} \partial_{\nu} \chi_{r 2}\right) \\
& -m_{\phi}^{2} \phi^{*} \phi-\frac{\lambda}{2}\left(\phi^{*} \phi\right)^{2}-m_{m 1}^{2} \chi_{m 1}^{*} \chi_{m 1} \\
& -\frac{1}{2} m_{m 2}^{2} \chi_{m 2}^{2}-m_{r 1}^{2} \chi_{r 1}^{*} \chi_{r 1}-\frac{1}{2} m_{r 2}^{2} \chi_{r 2}^{2}-\mu_{m} \phi^{*} \chi_{m 1} \chi_{m 2} \\
& \left.-\mu_{m}^{*} \chi_{m 1}^{*} \phi \chi_{m 2}-\mu_{r} \phi^{*} \chi_{r 1} \chi_{r 2}-\mu_{r}^{*} \chi_{r 1}^{*} \phi \chi_{r 2}\right\}
\end{aligned}
$$

Here $\phi, \chi_{m 1}, \chi_{r 1}$ are complex scalars while $\chi_{m 2}, \chi_{r 2}$ are real scalars. These are the particles in the two body scattering process in Fig. 1, namely, $\chi_{m 1}, \chi_{m 2}$ denote the incoming matter particles with momenta $p_{1}, p_{2}$ while $\chi_{r 1}, \chi_{r 2}$ denote the outgoing radiation particles with momenta $p_{3}, p_{4}$, and $\phi$ denotes the on-shell intermediate state particle in the figure.

The Klein-Gordon equation for $\phi$ is obtained from (56) as

$$
\begin{aligned}
& \frac{\partial^{2} \phi}{\partial t^{2}}+3 H \frac{\partial \phi}{\partial t}-\frac{c^{2}}{a^{2}} \Delta \phi+\frac{m_{\phi}^{2} c^{4}}{\hbar^{2}} \phi \\
& \quad+c^{2} \lambda \phi\left(\phi^{*} \phi\right)+c^{2} \mu_{m} \chi_{m 1} \chi_{m 2}+c^{2} \mu_{r} \chi_{r 1} \chi_{r 2}=0
\end{aligned}
$$

where $\Delta$ is the 3-dimensional Laplace operator, we have assumed the metric to be the spatially flat case of (1) i.e. we have assumed the perturbations of $\phi$ due to its motion be negligible with respect to the background, and we have introduced $c$ and $h$ into the equation that has been taken as $c=\hbar=1$ in (56) to see the reduction of (57) to Schrödinger equation in the non-relativistic limit more clearly.

To obtain the non-relativistic limit of (57) (to compare this case with the standard literature at atomic scales where cosmic expansion is not considered) we assume that the second term is negligible with respect to the others. One may neglect the second term provided that $\frac{m_{\phi} c^{2}}{h} \gg 3 H$ (that may be seen more easily after transforming $\phi$ to $\psi$ as given below). Consider the transformation $\phi=e^{-i \frac{m_{\phi} c^{2}}{\hbar} t} \psi$. Note that this transformation essentially subtracts the rest mass energy from the total energy of $\phi$. If $\phi$ is non-relativistic for a long time then its time evolution should be slow. These together, in turn, imply $\left|\frac{\ddot{\psi}}{\dot{\psi}}\right| \ll \frac{2 M_{\phi} c^{2}}{\hbar}$. Then (57) reduces to

$$
\begin{aligned}
& -i \hbar \dot{\psi}-\frac{\hbar^{2}}{2 a^{2} m_{\phi}} \Delta \psi+\tilde{\lambda} \psi\left(\psi^{*} \psi\right)+\tilde{\mu}_{m} \tilde{\chi}_{m 1} \chi_{m 2} \\
& +\tilde{\mu}_{r} \tilde{\chi}_{r 1} \chi_{r 2}=0 .
\end{aligned}
$$

Here $\psi=e^{i \frac{m_{\phi} c^{2}}{h} t} \phi, \tilde{\chi}_{m 1}=e^{i \frac{m_{\phi} c^{2}}{h} t} \chi_{m 1}, \tilde{\chi}_{r 1}=e^{i \frac{m_{\phi} c^{2}}{h} t} \chi_{r 1}$, $\tilde{\lambda}=\frac{h^{2}}{2 m_{\phi}} \lambda, \tilde{\mu}_{m}=\frac{h^{2}}{2 m_{\phi}} \mu_{m}, \tilde{\mu}_{r}=\frac{h^{2}}{2 m_{\phi}} \mu_{r}$. Eq. (58) is the Gross-Pitaevskii equation [23,24] for the metric in (1) where the trapping potential is zero and there is an external source term [25], namely, $\mu_{m} \tilde{\chi}_{m 1} \chi_{m 2}+\mu_{r} \tilde{\chi}_{r 1} \chi_{r 2}$. Note that Gross-Pitaevskii equation is the equation for a particle in a condensate, so it is the equation for the condensate state since all particles are in the same state in a Bose-Einstein condensate. The external source term in (58) accounts for the conversion of $\chi_{m 1}, \chi_{m 2}$ to $\psi$ and the decay of $\psi$ to $\chi_{r 1}$ and $\chi_{r 2}$.

We let the coupling constants $\mu_{m}$ and $\mu_{r}$ satisfy $\mu_{m} \gg \mu_{r}$ since we want a quick formation and a long lifetime for the condensate to simplify the situation. A short condensate formation time together with the condition that $\chi_{m 1}, \chi_{m 2}$ are non-relativistic and the mass of $\phi$ being comparable to those of $\chi_{m 1}, \chi_{m 2}$ guarantees smallness of any entropy production through the condensation process $[15,26]$. To be specific; the short duration of the condensation and small velocities guarantee smallness of any work done during the process, and the non-relativistic nature of $\chi_{m 1}, \chi_{m 2}, \phi$ guarantees the smallness of any heat production, so any probable entropy production due to mismatch of these terms will be small. In fact in the case of Bose-Einstein condensation in atomic gases the formation time of the condensate is very small compared to its lifetime [27-29]. Note that the condensates described by Gross-Pitaevskii equation for $\lambda>0$ correspond to an ideal case at $T=0$ where there are no inelastic two and three body collisions in the condensate that limits its life-time [29], hence a condensate described by Gross-Pitaevskii equation form more easily and is stable i.e. has an infinite lifetime. Therefore the assumption of a short time for the formation of the condensate in this case is a reasonable assumption. Moreover we require a long lifetime to obtain a simple model compatible with the present energy densities of dark energy and dark matter as we will see at the end of this sub-subsection. This requirement is easily satisfied provided that we take $\mu_{r}$ sufficiently small. The Eqs. (56) 
and (58) are similar to those in [19] except the additional scalars $\chi_{m i}, \chi_{r i}, i=1,2$; hence the presence of an external source term in this case. We let $m_{m 1}^{2} \chi_{m 1}^{*} \chi_{m 1} \gg \mu_{m} \chi_{m 1}^{*} \phi \chi_{m 2}, m_{m 2}^{2} \chi_{m 2}^{*} \chi_{m 2} \gg$ $\mu_{m} \chi_{m 1}^{*} \phi \chi_{m 2}$ so that one may consider $\chi_{m 1}, \chi_{m 2}$ as (almost) free particles, and in their non-relativistic limits their equations of state become (almost) zero, so considering them as matter particles is insured. In a similar way we let $m_{r 1}^{2} \chi_{r 1}^{*} \chi_{r 1} \gg \mu_{m} \chi_{r 1}^{*} \phi \chi_{r 2}$, $m_{r 2}^{2} \chi_{r 2}^{*} \chi_{r 2} \gg \mu_{m} \chi_{r 1}^{*} \phi \chi_{r 2}$ so that one may consider $\chi_{r 1}, \chi_{r 2}$ as (almost) free particles, and hence we may consider them as radiation provided that and $m_{r 1}^{2} \simeq 0, m_{r 2}^{2} \simeq 0$. Another important difference between [19] and this one is that [19] takes $\lambda<0$ while we take $\lambda>0$ which is the case in the original Gross-Pitaevskii equation. This makes the model simpler since the condensate in this case becomes stable (in the absence of the $\phi^{*} \chi_{1 r} \chi_{2 r}$ term) while in [19] the condensate experiences few collapse and reformation cycles. In fact the condensate in this case (even in the presence of the $\phi^{*} \chi_{1 r} \chi_{2 r}$ term) is rather stable since we take $\mu_{r}$ very small. Another potential point that makes the condensate more stable is the use of complex scalars i.e. charged scalars that makes the condensate more stable than a naive expectation [15]. We assume that the particle number density is sufficiently high enough so that the de Broglie wave length $\lambda_{d B}=\sqrt{\frac{2 \pi h^{2}}{m k T}}$ is larger than the mean separation $n^{-\frac{1}{3}}$ between the particles. This condition may be converted to an upper bound on the temperature of $\phi$ 's

$T<T_{c}=\frac{2 \pi \hbar^{2} n^{\frac{2}{3}}}{k\left(\zeta\left(\frac{3}{2}\right)\right)^{\frac{2}{3}} m_{\phi}}$

where $k$ is the Boltzmann constant, $n$ is the number density. In the case where the $\phi$ 's were in thermal equilibrium with baryonic matter and radiation at some initial time, Eq. (59) puts an upper bound on $m_{\phi}$ while there is no bound on $m_{\phi}$ in the case where $\phi$ 's never had a thermal equilibrium with baryonic matter and radiation. In the case of non-relativistic $\phi$ 's the temperature of $\phi$ 's remain below $T_{c}$ at later times once it is below $T_{c}$ at some initial time in the past [19]. In this study we assume that $\phi$ 's are nonrelativistic and they had never thermal equilibrium with baryonic matter and radiation in the past.

After the basic elements of condensate formation for (56), now we are ready to derive its results at macroscopic level by using the general analysis we have given. In another words we will relate the microscopic view (i.e. the particle physics view) of the condensate given above to its macroscopic view at the level of energy densities in the light of the analysis we have given before this sub-subsection. The evolution of the energy densities may be derived from (6), (23), and (38), (26), as

$$
\begin{aligned}
& H^{2}=\frac{8 \pi G}{3}\left(\rho_{m}+\rho_{\phi}+\rho_{r}\right) \\
& \dot{\rho}_{m}+3 H \rho_{m}=-\frac{d \tilde{\rho}_{m}}{d t}=-\beta(a(t))^{r-1} \rho_{m}^{2} \\
& \dot{\rho}_{\phi}+3 H\left(1+\omega_{\phi}\right) \rho_{\phi}=\frac{d \tilde{\rho}_{m}}{d t}-\Gamma \rho_{\phi} \\
& \quad=\beta(a(t))^{r-1} \rho_{m}^{2}-\Gamma \rho_{\phi} \\
& \dot{\rho}_{r}+4 H \rho_{m}=\frac{d \tilde{\rho}_{r}}{d t}=\Gamma \rho_{\phi}
\end{aligned}
$$

where $\rho_{m}$ consists of $\chi_{m 1}$ and $\chi_{m 2}, \rho_{r}$ consists of $\chi_{r 1}$ and $\chi_{r 2}$, and $\rho_{\phi}$ consists of the $\phi$ particles. Here we do not make any distinction between the resonance particles produced as the intermediate state of the 4-point function in Fig. 1 and the particles in the condensate because we assume that the condensate is formed almost simultaneously after the formation of the resonance particles as we have mentioned before. The Eqs. (61), (62) somewhat differ from those of [19] since the $\frac{d \tilde{\rho}_{m}}{d t}$ term in the above equations is proportional to $\rho_{m}$ in [19] while it is proportional to $\rho_{m}^{2}$ here. Taking $\frac{d \tilde{\rho}_{m}}{d t}$ proportional to $\rho_{m}^{2}$ (due to (38) seems more correct in the view that Bose-Einstein condensation involves interaction of particles in a Bose gas rather than their decay (where decay rate would be proportional to the energy density of the decaying particles). [19] also considers a possible dependence of the decay widths on the scale factor in an ad hoc way. While the dependence of $\frac{d \tilde{\rho}_{m}}{d t}$ on scale factor in this study and in [19] are similar their origins are different. The origin of the dependence of $\frac{d \tilde{\rho} m}{d t}$ in [19] is through $\Gamma$ 's while in our case it results from the propagator part of the cross section, not through $\Gamma$ 's. We have found that dependence of the self energies on the momentum in perturbative quantum field theory suggests that the leading order contribution to $\Gamma$ is independent of scale factor. Moreover the explicit form of $\rho_{m}$ in terms of the cross section is derived in this study.

One may see the general lines of the formation of the condensate by following a procedure similar to that of [19]. Eq. (62) be written as

$\dot{\rho}_{\phi}+6 H\left[\dot{\phi}^{*} \dot{\phi}+\frac{1}{a^{2}}\left(\vec{\nabla} \phi^{*}\right) \cdot(\vec{\nabla} \phi)\right]=\beta[a(t)]^{r-1} \rho_{m}^{2}-\Gamma \rho_{\phi}$.

Here we have used $\rho_{\phi}+p_{\phi}=2\left[\dot{\phi}^{*} \dot{\phi}+\frac{1}{a^{2}}\left(\vec{\nabla} \phi^{*}\right) \cdot(\vec{\nabla} \phi)\right]$. Note that in a case where $\phi$ is homogeneous we would have $\vec{\nabla} \phi=0$ while here we take it non-zero at initial times of the formation of the condensate (although the background is taken as homogeneous). Otherwise there will be no condensate formation starting from $\rho_{\phi}=0$ at initial time $t_{1}$ since

$\rho_{\phi}=\dot{\phi}^{*} \dot{\phi}+\frac{1}{a^{2}}\left(\vec{\nabla} \phi^{*}\right) \cdot(\vec{\nabla} \phi)+V$

implies

$\phi\left(t_{1}\right)=0, \dot{\phi}\left(t_{1}\right)=0, \vec{\nabla} \phi\left(t_{1}\right)=0$

because

$V=m_{\phi}^{2} \phi^{*} \phi+\frac{\lambda}{2}\left(\phi^{*} \phi\right)^{2}+\mu_{m} \phi^{*} \chi_{m 1} \chi_{m 2}$

$+\mu_{r} \phi^{*} \chi_{r 1} \chi_{r 2}+$ h.c.

(where h.c. implies taking the Hermitian conjugate of the proceeding terms) is positive for all values of $\phi$ since we take all coefficients positive. (In fact even in the case where $\mu_{m}, \mu_{r}$ are negative $V$ is still positive since we take the corresponding terms very small compared to the rest of the terms). These together with Eq. (57) imply that we should have

$\Delta \phi\left(t_{1}\right)>0$ and $\frac{\partial^{2} \phi\left(t_{1}\right)}{\partial t^{2}}>0$.

During the formation of the condensate one must have $\dot{\rho}_{\phi}>$ 0 . Therefore Eq. (64) implies that during the formation of the condensate one has one must have

$$
\begin{gathered}
\beta(a(t))^{r-1} \rho_{m}^{2}-\Gamma \rho_{\phi}>6 H\left(\rho_{\phi}-V\right) \\
=6 H\left(|\dot{\phi}|^{2}+\frac{1}{a^{2}}|\vec{\nabla} \phi|^{2}\right)>0
\end{gathered}
$$

$\dot{\phi}, \vec{\nabla} \phi$ start with the values given in (66) and increase because of (68) till the bound in (69) is saturated. Eq. (57) implies that first $\frac{\partial^{2} \phi}{\partial t^{2}}$ becomes zero i.e. $\dot{\phi}$ reaches its maximum value while $\vec{\nabla} \phi$ keeps rising i.e. still $\Delta \phi>0 . \vec{\nabla} \phi$ keeps rising till the bound in (69) is saturated once again. (Note that, as the right hand side of the inequality in (69) increases its left hand side decreases by time since the energy density of $\rho_{m}$ gets smaller, that of $\rho_{\phi}$ gets larger.) Then $\Delta \phi$ changes sign and $|\vec{\nabla} \phi|^{2}$ decreases too till it eventually 
becomes zero, so the right hand-side (69) becomes zero. Finally the left-hand side of (69) becomes zero too by evolution of the energy densities, and the final values of $\rho_{m}$ and $\rho_{\phi}$ are reached (provided that the decay of $\phi$ is neglected i.e. the term with coefficient $\mu_{r}$ is neglected since it very small). Therefore one may get a rough picture of the evolution of the energy densities.

Now let us see if this model is compatible with the present day energy densities, at least, at an order of magnitude level. We will identify the condensate by dark energy and the leftover part of $\rho_{m}$ after formation of the condensate by dark matter. We will neglect the contributions due to baryonic matter and (the observable) radiation for simplicity. We take the time that passed since the start of the condensation $t_{1}$ till the present time $t_{0}$ be much smaller than the lifetime of the condensate i.e. we let $\left(t_{0}-t_{1}\right) \ll \Gamma^{-1}$ to ensure that the energy density of $\chi_{r 1}$ and $\chi_{r 2}$ is below the observational bounds on dark radiation [30]. This condition is expressed in a more concrete form in the next equation. To derive that equation we make use of the following approximations and equations: We take $\rho_{\phi}$ almost constant from $t_{1}$ to $t_{0}$, and make use of (3) and (27) (where $\rho_{R}=\rho_{\phi}$ ). We approximate the integral in (27) as the sum of two parts; the first part being from $t_{1}$ to $t_{q}$ where we assume the universe was wholly matter dominated and from $t_{q}$ to $t_{0}$ where we assume that the universe was wholly $\rho_{\phi}$ dominated which is a rough approximation to the evolution of the universe. We neglect the radiation dominated era because we neglect the contribution of (the observable) radiation in this study. (In fact even we had included the contribution of the usual observable radiation its effect would turn out to be negligible in the integral given below.) Then (27) and (3) imply

$$
\begin{aligned}
\frac{\frac{\Omega_{\gamma}}{10}}{\Omega_{D E}} & \simeq 10^{-5}>\frac{\Omega_{r}}{\Omega_{D E}}=\frac{\rho_{r}\left(t_{0}\right)}{\rho_{\phi}\left(t_{0}\right)} \simeq \Gamma \int_{t_{1}}^{t_{0}} a^{4}\left(t^{\prime}\right) \theta\left(t-t^{\prime}\right) d t^{\prime} \\
& \simeq \frac{\Gamma}{H_{0}}\left[\int_{a\left(t_{1}\right)}^{a\left(t_{q}\right)} \frac{\left(a^{\prime}\right)^{\frac{9}{2}}}{\sqrt{\Omega_{D M}}} d a^{\prime}+\int_{a\left(t_{q}\right)}^{a\left(t_{0}\right)} \frac{\left(a^{\prime}\right)^{3}}{\sqrt{\Omega_{D E}}} d a^{\prime}\right] \\
& \simeq 0.3 \frac{\Gamma}{H_{0}} \simeq 0.3 t_{0} \Gamma
\end{aligned}
$$

where we used the fact that the present energy density of a radiation component other than photons and neutrinos, $\rho_{r}$ is, at most, at the level of a tenth of the energy density of photons $\rho_{\gamma}$ [30]; $\rho_{D E}$ is the present energy density for dark energy; $\Omega$ 's denote the corresponding density parameters, and we have used the numerical values of the density parameters in [9] where $\Omega_{D E}=\Omega_{\Lambda}$ and that the time for the beginning of the dark energy dominated era is at about the redshift $z=0.65$ i.e. at $a\left(t_{q}\right)=0.6$. One may obtain a similar result by using a more heuristic approach as well: Provided that $\Gamma\left(t_{0}-t_{1}\right) \ll 1$, the ratio of the energy density of the decayed part of the condensate to its energy density at present $\frac{\rho_{r 0}}{\rho_{D E 0}}$ roughly satisfies

$\frac{\frac{\Omega_{\gamma}}{10}}{\Omega_{D E}} \simeq 10^{-5}>\frac{\rho_{r 0}}{\rho_{D E 0}}=\frac{1-e^{-\Gamma\left(t_{0}-t_{1}\right)}}{e^{-\Gamma\left(t_{0}-t_{1}\right)}} \simeq \Gamma\left(t_{0}-t_{1}\right)$.

In the notation of the preceding sub-subsection we have $t_{2}<t_{0}$ $\ll t_{3}$. If $\phi$ never had a thermal equilibrium with baryonic matter and photons then the condensation must have started before matter dominated era if we want to mimic $\Lambda$ CDM (because of its observational success). On the other hand, if $\phi$ had thermal equilibrium with baryonic matter and photons in the past then $\phi$ must have decoupled from baryonic matter and radiation before the time of nucleosynthesis to keep the successful nucleosynthesis scenario of the standard model intact. In either case $t_{0}-t_{1}$ must be in the order of the age of the universe i.e. in the order of $10^{17}$ seconds. Hence (70) or (71) implies that $\frac{1}{\Gamma}>10^{22} \mathrm{~s}$. In the light of these observations we derive a rough lower bound on the value of the production cross section of $\phi$ 's by using (69). Since $\frac{1}{\Gamma}>10^{22}$ $\mathrm{s}$, and after the end of the formation process of the condensate (as discussed after (69)) we have $\beta(a(t))^{r-1} \rho_{m}^{2}-\Gamma \rho_{\phi}=0$, we obtain

$$
\begin{aligned}
\frac{1}{10^{22} \mathrm{~s} \times \beta}<\frac{\Gamma}{\beta} & =\frac{\rho_{m}\left(t_{0}\right)}{\rho_{\phi}\left(t_{0}\right)} \rho_{m}\left(t_{0}\right)=\simeq\left(\frac{0.26}{0.69}\right) \times 0.26 \rho_{\text {crit }} \\
& \simeq 0.1 \rho_{\text {crit }} .
\end{aligned}
$$

Here $\rho_{\text {crit }}$ is the critical energy density at present. After using (72) and the expression for $\beta$ in (38) and the numerical value of $\rho_{\text {crit }}$ we may write

$\beta=\frac{\beta^{\prime} \sigma\left(t_{1}\right) v\left(t_{1}\right)}{E a\left(t_{1}\right)}>\left(\frac{16}{9}\right) \times 10^{-25}(\mathrm{eV})^{-1}(\mathrm{~s})^{-1}$

i.e.

$\frac{\sigma\left(t_{1}\right)}{\mathrm{cm}^{2}}>\left(\frac{E}{\mathrm{eV}}\right)\left(\frac{\mathrm{cm} / \mathrm{s}}{v\left(t_{1}\right)}\right) \frac{a\left(t_{1}\right)}{\beta^{\prime}}\left(\frac{16}{9} \times 10^{-25}\right)$.

It is evident from (74) that one can produce the values of the present dark matter and dark energy density parameters $\Omega_{D M} \simeq$ 0.26 and $\Omega_{D E} \simeq 0.69$ for a wide range of cross sections. For example if we take $v\left(t_{1}\right)=\frac{1}{3} \times 10^{-4} c, a\left(t_{1}\right)=10^{-10}, \beta^{\prime}=\frac{1}{10},\left(\frac{E}{\mathrm{eV}}\right)=1$ then we find $\sigma\left(t_{1}\right)>\frac{16}{9} \times 10^{-42} \mathrm{~cm}^{2}$, and $\sigma\left(t_{1}\right)>10^{-26} \mathrm{~cm}^{2}$ if $\left(\frac{E}{\mathrm{eV}}\right)=10^{9}, a\left(t_{1}\right)=10^{-3}$ and all other parameters kept the same. We may also check if the formula we have derived for $\rho_{R}$ in this section is consistent with a constant value for $\rho_{\phi}$ at present provided that we adopt the values of parameters given above. In this case one cannot directly use the results of the preceding subsubsection because in this case $\gamma=\frac{\Gamma}{H} \ll 1$ while in (51) (or in (B.16)) we had taken $\gamma=3$ to simplify the integrals for $\rho_{r}$. Instead we should go back to the original equation in (B.11) and make use of the fact that $\frac{\rho_{m 0} \beta}{\xi_{i}} \ll 1, i=1,2$ in this case since $\xi_{i}$ are in the order of $H_{0}$. Therefore $\left(\frac{d \tilde{\rho}_{m}(t)}{d t}\right)_{2} \simeq \theta\left(t-t_{2}\right) \rho_{m 0}^{2} \beta$. Then we insert this value in (B.12) while we keep the exponential factor, and take the integral, and finally we use $\gamma=\frac{\Gamma}{H} \ll 1$. Hence in this case $C_{R}$ is essentially constant at present, so the same is true for $\rho_{R}=\rho_{\phi}$.

\subsection{Some additional remarks for an intermediate state resonance}

In the preceding subsection we have given a particle physics description of an intermediate state with negative equation of state in the conversion of matter to radiation in terms of resonance particles that form a Bose-Einstein condensate. We have also considered a concrete model in this context to see the situation more explicitly. However such a specific model is not enough to see all aspects of these types of models. We have neglected the era for formation of the condensate since it is expected to be very small compared to its lifetime and to be able focus on the more relevant points. A similar situation is true for the era of the decay of the condensate since this era corresponds to far future in our model, so phenomenologically is less interesting. Although the study of these eras in detail is not essential in the context of the model we have considered for late time cosmic acceleration it would be relevant in models of inflation, especially in those where the condensate decays into usual radiation rather than to dark radiation. Therefore in the following paragraphs we give the overall picture in terms of some simple generic hypothetical energy distribution to see the overall picture more clearly.

After adding (6), (7), (22), and using (25) one obtains

$\dot{\rho}(t)+3 H\left[1+\frac{1}{3} \frac{\rho_{r}(t)}{\rho(t)}+\omega_{R} \frac{\rho_{R}(t)}{\rho(t)}\right] \rho(t)=0$

$\rho(t)=\rho_{m}(t)+\rho_{r}(t)+\rho_{R}(t)$.

Now we consider some specific cases in the light of the Eq. (75). Note that $\frac{\rho_{R}}{\rho}$ may be close to 1 , especially if we identify $\rho_{R}$ by a 
resonance or a condensate formed as an intermediate state in the transition of matter to radiation as in the second graph in Fig. 1. Therefore the effect of $\rho_{R}$ may be sizeable for some time interval in the evolution of the universe. Moreover, since $\rho_{R}$ corresponds to some intermediate state in the conversion of matter to radiation it must be localized in time. In the light of these observations we consider some possible forms for $\rho_{R}$. This will give us an idea about the possible effects of conversion of matter to radiation (or vice versa) on the expansion rate of the universe. In fact, to have a concrete, realistic picture one needs to calculate the cross sections and the rates of different such process in an expanding universe for different possible scenarios. We leave this ambitious program to detailed studies in future. In this subsection we give an idea on the range of possibilities by considering some hypothetical choices of $\rho_{R}$ 's that are localized in time, and this will be sufficient for our purpose. First consider the example, $\rho_{R}=\sigma\left[1-\tanh ^{2} \beta\left(a-a_{i}\right)\right]$ where $\sigma, \beta$ are some constants and $a_{i}$ denotes the scale factor where $\rho_{R}$ is maximum. Then $\frac{\dot{\rho}_{R}}{\rho_{R}}=-\left[2 \beta \dot{a} \tanh \beta\left(a-a_{i}\right)\right]$. After using (24) one gets

$\omega_{R}+\Delta \omega_{R}=-\frac{\dot{\rho_{R}}}{3 H \rho_{R}}-1=\frac{2}{3} \beta a \tanh \beta\left(a-a_{i}\right)-1$.

One notices that

$$
\begin{aligned}
& \omega_{R}+\Delta \omega_{R} \rightarrow-1 \text { as } a \rightarrow 0 \text { or } a \rightarrow a_{i}, \\
& \omega_{R}+\Delta \omega_{R} \leq-1 \text { when } 0<a<a_{i} \text { and } \\
& \omega_{R}+\Delta \omega_{R} \geq-1 \text { when } a>a_{i} \\
& \omega_{R} \rightarrow \infty \text { as } a \rightarrow \infty, \text { and } \\
& \omega_{R}+\Delta \omega_{R} \simeq 0.28 \text { when }\left(a-a_{i}\right) \beta \simeq 2 .
\end{aligned}
$$

Next consider another example, $\rho_{R}=\frac{\sigma}{1+\beta\left(a-a_{i}\right)^{2}}$. Then $\frac{\dot{\rho}_{R}}{\rho_{R}}=$ $\frac{2 \beta \dot{a}\left(a-a_{i}\right)}{\left[1+\beta\left(a-a_{i}\right)^{2}\right]^{2}}$. After using (24) one gets

$\omega_{R}+\Delta \omega_{R}=-\frac{\dot{\rho}_{R}}{3 H \rho_{R}}-1=\frac{2 \beta a\left(a-a_{i}\right)}{3\left[1+\beta\left(a-a_{i}\right)^{2}\right]}-1$.

We find that

For $a=\frac{a_{i}}{2} \omega_{R}+\Delta \omega_{R}=\left(\omega_{R}+\Delta \omega_{R}\right)_{\min }=-\frac{2 \beta\left(\frac{a_{i}}{2}\right)^{2}}{3\left[1+\beta\left(\frac{a_{i}}{2}\right)^{2}\right]}-1$

and $\omega_{R}+\Delta \omega_{R}>\left(\omega_{R}+\Delta \omega_{R}\right)_{\min }$

$\omega_{R}+\Delta \omega_{R} \rightarrow-1$ as $a \rightarrow 0$ or $a \rightarrow a_{i}$ and

$\omega_{R}+\Delta \omega_{R} \rightarrow-\frac{1}{3}$ as $a \rightarrow \infty$

where the subindex min denotes the minimum value of $\omega_{R}+\Delta \omega_{R}$.

In both examples we have $\omega_{R}+\Delta \omega_{R}=-1$ at $a=a_{i}$ and $\omega_{R}+\Delta \omega_{R}<-1$ for $a<a_{i}, \omega_{R}+\Delta \omega_{R}>-1$ for $a>a_{i}$. This is not surprising, it is just a result of the requiring $\rho_{R}$ be localized. Therefore most of the general lines of the above examples are valid in all cases provided that $\rho_{R}$ is localized in time. Note that both in this set-up and in the standard model of cosmology, the universe is radiation dominated at the time just after the time of creation of radiation. Moreover $\frac{\rho_{R}}{\rho} \simeq 1$ when $a \simeq a_{i}$. Thus in a model of inflation in this context one may take the $\omega_{R}+\Delta \omega_{R}$ values obtained for the times close to the time $a=a_{i}$ as a sufficiently realistic of the universe for the time just before creation of radiation. On the other hand the same is not true for other times e.g. for much later times since, at much later times, the universe almost exhaustively is composed of components other than radiation e.g. matter (and possibly dark energy as well). Therefore a more realistic modification of the content of the universe is needed to extend this scheme to account for early time cosmic accelerated expansion. However regarding dark energy even these simple examples may give some insight to the problem: A residual effect of the $\rho_{R}$ that survives may have some effect for later times. Although tiny, $\rho_{R}$ may still have a tail for large scale factor, $a(t)$. The effect of this tail is determined by how well $\rho_{R}$ is localized, and how big $\rho_{R}$ and $\omega_{R}+\Delta \omega_{R}$ are. In fact such a model is considered in literature [21].

\section{Conclusion}

In this paper we have studied, as a sub-case of two interacting energy densities, if the accelerated cosmic expansion may be induced by conversion of extremely non-relativistic particles to radiation. We have seen that cosmic accelerated expansion cannot be obtained in conversion between matter and radiation through instantaneous interactions. In fact thermodynamical studies give a similar conclusion [31]. It seems that the only way to obtain cosmic accelerated expansion by particle physics interactions is through some intermediate state with a negative equation of state that forms during the conversion. It seems difficult to obtain the present cosmic accelerated expansion wholly through the usual particle physics interactions in this way since the localization scales of corresponding $\rho_{R}$ 's for the usual particle physics processes are at the order of atomic scales i.e. at scales much smaller than the cosmological scales. Even when they have such an effect, these interactions will first accelerate the universe and then decelerate it in the time scale of the interaction time (which is smaller than $\sim 10^{-8} \mathrm{~s}$ ), hence the net effect would be zero. This type of interactions may be relevant cosmologically only at early times (if they involve the usual particles) provided that a significant redshift takes place during their interaction time e.g. during the lifetime of the resonance particle. A very early time acceleration may be induced by fast out of equilibrium processes as those given in Fig. 1 provided an intermediate state with $\omega<0$ forms. Present cosmic accelerated expansion may be obtained in this way only if the life time of the resonance condensate has a cosmologically relevant time scale. Although we have considered such a toy model in this study, in order to entertain these possibilities in detail one needs to study different specific models in more detail along the lines given in this paper and confront it with observational data which is beyond the scope of this study that aims to seek the degree of possibility of obtaining the late time and the early time accelerated expansions of the universe in this way. Specific models along these lines where different options for $\rho_{R}$ and $\frac{\tilde{\rho}(t)}{d t}$ are specified and their theoretical origins discussed and whose the results are confronted with observational data may be considered in future.

\section{Acknowledgments}

I would like to thank Professor Luca Amendola for pointing out a crucial calculational error in the draft version of this paper. I would also like to thank Professor Masahiro Morikawa for his valuable detailed comments regarding their papers related to the use of Bose-Einstein condensation for cosmic accelerated expansion.

\section{Appendix A. Details of the derivations of (4), (5), (6), (7)}

Let at time $t<t_{1}$ the energy density of the universe, $\rho$ consists of matter (i.e. dust) and radiation, that is,

$\rho=\rho_{m}+\rho_{r}$

$\rho_{m}=\frac{\rho_{m 0}}{[a(t)]^{3}}, \quad \rho_{r}=\frac{\rho_{r 0}}{[a(t)]^{4}}$.

Assume that at $t_{1}<t<t+\Delta t$ (where $\Delta t$ is very small) some energy density $(\Delta \tilde{\rho})_{1}$ is transferred from either of the dust or the radiation to the other, say, from the dust to the radiation e.g. through some decay or other particle physics process such as in 
Fig. 1. Then the new energy density becomes

$$
\begin{aligned}
\rho(t)= & \left\{\frac{\rho_{m 0}}{[a(t)]^{3}}-(\Delta \tilde{\rho})_{1} \theta\left[t-\left(t_{1}+\Delta t\right)\right]\left[\frac{a\left(t_{1}+\Delta t\right)}{a(t)}\right]^{3}\right\} \\
& +\left\{\frac{\rho_{r 0}}{[a(t)]^{4}}+(\Delta \tilde{\rho})_{1} \theta\left[t-\left(t_{1}+\Delta t\right)\right]\left[\frac{a\left(t_{1}+\Delta t\right)}{a(t)}\right]^{4}\right\} \\
= & \frac{\rho_{m 0}-(\Delta \tilde{\rho})_{1} \theta\left[t-\left(t_{1}+\Delta t\right)\right]\left[a\left(t_{1}+\Delta t\right)\right]^{3}}{[a(t)]^{3}} \\
& +\frac{\rho_{r 0}+(\Delta \tilde{\rho})_{1} \theta\left[t-\left(t_{1}+\Delta t\right)\right]\left[a\left(t_{1}+\Delta t\right)\right]^{4}}{[a(t)]^{4}}
\end{aligned}
$$

where $\theta(t)$ is the Heaviside function (i.e. unit step function) with $\theta(t)=0$ if $t<0$, and $\theta(t)=1$ if $t \geq 0$. Next assume that at $t_{1}+\Delta t<t<t_{1}+2 \Delta t$ some other energy density $(\Delta \tilde{\rho})_{2}$ is transferred from the dust to the radiation, and so on. Hence at $t=t_{1}+n \Delta t$ the energy density is

$\rho(t)=\frac{\rho_{M \Delta}}{[a(t)]^{3}}+\frac{\rho_{r \Delta}}{[a(t)]^{4}}$

where

$$
\begin{aligned}
\rho_{M \Delta}= & \rho_{m 0}-(\Delta \tilde{\rho})_{1} \theta\left[t-\left(t_{1}+\Delta t\right)\right] a^{3}\left(t_{1}+\Delta t\right) \\
& -(\Delta \tilde{\rho})_{2} \theta\left[t-\left(t_{1}+2 \Delta t\right)\right] a^{3}\left(t_{1}+2 \Delta t\right) \cdots \\
& -(\Delta \tilde{\rho})_{n} \theta\left[t-\left(t_{1}+n \Delta t\right)\right] a^{3}\left[\left(t_{1}+n \Delta t\right)\right] \\
\rho_{r \Delta}= & \rho_{r 0}+(\Delta \tilde{\rho})_{1} \theta\left[t-\left(t_{1}+\Delta t\right)\right] a^{4}\left(t_{1}+\Delta t\right) \\
& +(\Delta \tilde{\rho})_{2} \theta\left[t-\left(t_{1}+2 \Delta t\right)\right] a^{4}\left(t_{1}+2 \Delta t\right) \cdots \\
& +(\Delta \tilde{\rho})_{n} \theta\left[t-\left(t_{1}+n \Delta t\right)\right] a^{4}\left[\left(t_{1}+n \Delta t\right)\right] .
\end{aligned}
$$

As $\Delta t \rightarrow 0,(\Delta \tilde{\rho})_{i} \rightarrow 0(i=1,2, \ldots)$ the energy density in (A.4) at $t=t_{2}>t_{1}$ becomes

$$
\begin{aligned}
& \rho\left(t_{2}\right)=\rho_{m}\left(t_{2}\right)+\rho_{r}\left(t_{2}\right) \\
& \rho_{m}\left(t_{2}\right)=\frac{C_{m}\left(t_{2}\right)}{\left[a\left(t_{2}\right)\right]^{3}}, \quad \rho_{r}\left(t_{2}\right)=\frac{C_{r}\left(t_{2}\right)}{\left[a\left(t_{2}\right)\right]^{4}}
\end{aligned}
$$

where

$$
\begin{aligned}
C_{m}( & \left.t_{2}\right)=\rho_{m 0}-\lim _{\Delta t \rightarrow 0, n \Delta t \rightarrow t_{2}-t_{1}}\left\{\left(\frac{(\Delta \tilde{\rho})_{1}}{\Delta t}\right)\right. \\
\times & \theta\left[t_{2}-\left(t_{1}+\Delta t\right)\right] a^{3}\left(t_{1}+\Delta t\right) \\
+ & \left(\frac{(\Delta \tilde{\rho})_{2}}{\Delta t}\right) \theta\left[t_{2}-\left(t_{1}+2 \Delta t\right)\right] a^{3}\left(t_{1}+2 \Delta t\right) \ldots \\
+ & \left.\left(\frac{(\Delta \tilde{\rho})_{n}}{\Delta t}\right) \theta\left[t_{2}-\left(t_{1}+n \Delta t\right)\right] a^{3}\left[\left(t_{1}+n \Delta t\right)\right]\right\} \\
= & \rho_{m 0}-\int_{t_{1}}^{t_{2}}\left(\frac{d \tilde{\rho}\left(t^{\prime}\right)}{d t^{\prime}}\right) a^{3}\left(t^{\prime}\right) \theta\left(t_{2}-t^{\prime}\right) d t^{\prime} \\
= & \rho_{m 0}-\int_{0}^{t_{2}-t_{1}}\left(\frac{d \tilde{\rho}(x)}{d x}\right)_{x=\left(t_{2}-u\right)} a^{3}\left(t_{2}-u\right) \theta(u) d u \\
C_{r}\left(t_{2}\right) & =\rho_{r 0}+\lim \Delta t \rightarrow 0, n \Delta t \rightarrow t_{2}-t_{1}\left\{\left(\frac{(\Delta \tilde{\rho})_{1}}{\Delta t}\right)\right. \\
& \times \theta\left[t_{2}-\left(t_{1}+\Delta t\right)\right] a^{4}\left(t_{1}+\Delta t\right) \\
& +\left(\frac{(\Delta \tilde{\rho})_{2}}{\Delta t}\right) \theta\left[t_{2}-\left(t_{1}+2 \Delta t\right)\right] a^{4}\left(t_{1}+2 \Delta t\right) \ldots \\
& \left.+\left(\frac{(\Delta \tilde{\rho})_{n}}{\Delta t}\right) \theta\left[t_{2}-\left(t_{1}+n \Delta t\right)\right] a^{4}\left[\left(t_{1}+n \Delta t\right)\right]\right\} \\
= & \rho_{r 0}+\int_{t_{1}}^{t_{2}}\left(\frac{d \tilde{\rho}\left(t^{\prime}\right)}{d t^{\prime}}\right) a^{4}\left(t^{\prime}\right) \theta\left(t_{2}-t^{\prime}\right) d t^{\prime} \\
\rho_{r}-t_{1} & \left(\frac{d \tilde{\rho}(x)}{d x}\right)_{x=\left(t_{2}-u\right)} a_{0}^{4}(t-u) \theta(u) d u .
\end{aligned}
$$

$$
\begin{aligned}
& \text { From (A.8) we see that (for } t>t_{1} \text { ) } \\
& \dot{\rho}_{m}(t)=-3 H \rho_{m}(t)+\frac{\dot{C}_{m}(t)}{[a(t)]^{3}} \\
& \dot{\rho}_{r}(t)=-4 H \rho_{r}(t)+\frac{\dot{C}_{r}(t)}{[a(t)]^{4}}
\end{aligned}
$$

where over dot denotes derivative with respect to t. Eqs. (A.13) and (A.14) imply that $\rho_{m}$ and $\rho_{r}$ satisfy the following equations

$\dot{\rho}_{m}(t)+3 H \rho_{m}(t)=\frac{\dot{C}_{m}(t)}{[a(t)]^{3}}$
$\dot{\rho}_{r}(t)+4 H \rho_{r}(t)=\frac{\dot{C}_{r}(t)}{[a(t)]^{4}}$.

We see that the energy-momentum tensors for the dust and the radiation in this case are conserved. They have source terms on the right-hand sides of (A.15) and (A.16). This is not surprising since there is an energy transfer from the dust to the radiation. If one adds (A.15) to (A.16) one obtains

$\dot{\rho}_{m}(t)+\dot{\rho}_{r}(t)+3 H\left[\rho_{m}(t)+\frac{4}{3} \rho_{r}(t)\right]=\frac{\dot{C}_{m}(t)}{[a(t)]^{3}}+\frac{\dot{C}_{r}(t)}{[a(t)]^{4}}$.

First calculate $\dot{C}_{m}(t)$, that may be found from

$\dot{C}_{m}(t)=\lim _{\Delta t \rightarrow 0} \frac{C_{m}(t+\Delta t)-C_{m}(t)}{\Delta t}$.

In the above formula we employ Eqs. (A.10) and (A.12) (rather than Eqs. (A.9) and (A.11)) not to deal with variation the $\theta$ function that may lead to ambiguity since we do not cover $t^{\prime}>t$ in the integration. Hence

$$
\begin{aligned}
C_{m}(t+\Delta t)= & \rho_{m 0}-\int_{0}^{t+\Delta t-t_{1}}\left(\frac{d \tilde{\rho}(x)}{d x}\right)_{x=(t+\Delta t-u)} \\
& \times a^{3}(t+\Delta t-u) \theta(u) d u \\
C_{r}(t+\Delta t)= & \rho_{r 0}+\int_{0}^{t+\Delta t-t_{1}}\left(\frac{d \tilde{\rho}(x)}{d x}\right)_{x=(t+\Delta t-u)} \\
& \times a^{4}(t+\Delta t-u) \theta(u) d u .
\end{aligned}
$$

Let us calculate these explicitly.

$$
\begin{aligned}
& \int_{0}^{t+\Delta t-t_{1}}\left(\frac{d \tilde{\rho}(x)}{d x}\right)_{x=(t+\Delta t-u)} a^{3}(t+\Delta t-u) \theta(u) d u \\
& =\int_{0}^{t-t_{1}}\left(\frac{d \tilde{\rho}}{d x}\right)_{x=(t+\Delta t-u)} a^{3}(t+\Delta t-u) \theta(u) d u \\
& \quad+\int_{t-t_{1}}^{t-t_{1}+\Delta t}\left(\frac{d \tilde{\rho}(x)}{d x}\right)_{x=(t+\Delta t-u)} a^{3}(t+\Delta t-u) \theta(u) d u
\end{aligned}
$$

For small $\Delta t$

$$
\begin{aligned}
& a^{3}(t+\Delta t-u)=\left[a(t-u)+\left(\frac{d a(x)}{d x}\right)_{x=t-u} \Delta t+\cdots\right]^{3} \\
& \simeq a^{3}(t-u)+3 a^{2}(t-u)\left(\frac{d a(x)}{d x}\right)_{x=t-u} \Delta t \\
& \left(\frac{d \tilde{\rho}(x)}{d x}\right)_{x=t+\Delta t-u} \simeq\left(\frac{d \tilde{\rho}(x)}{d x}\right)_{x=t-u}+\left(\frac{d^{2} \tilde{\rho}(x)}{d x^{2}}\right)_{x=t-u} \Delta t \\
& \int_{t-t_{1}}^{t-t_{1}+\Delta t}\left(\frac{d \tilde{\rho}}{d u}\right)(t+\Delta t-u) a^{3}(t+\Delta t-u) \theta(u) d u \\
& \simeq\left(\frac{d \tilde{\rho}}{d x}\right)_{x=t_{1}+\Delta t} a^{3}\left(t_{1}+\Delta t\right) \theta\left(t-t_{1}\right) \Delta t \\
& \simeq\left(\frac{d \tilde{\rho}}{d x}\right)_{x=t_{1}} a^{3}\left(t_{1}\right) \theta\left(t-t_{1}\right) \Delta t .
\end{aligned}
$$


Hence we find

$$
\begin{aligned}
& C_{m}(t+\Delta t)=\rho_{m 0}-\int_{0}^{t-t_{1}}\left\{\left[\left(\frac{d \tilde{\rho}(x)}{d x}\right)_{x=t-u}\right.\right. \\
& \left.+\left(\frac{d^{2} \tilde{\rho}(x)}{d x^{2}}\right)_{x=t-u} \Delta t\right] \\
& \left.\quad \times\left[a^{3}(t-u)+3 a^{2}(t-u)\left(\frac{d a(x)}{d x}\right)_{x=t-u} \Delta t\right] \theta(u) d u\right\} \\
& \quad-\left(\frac{d \tilde{\rho}(x)}{d x}\right)_{x=t_{1}} a^{3}\left(t_{1}\right) \theta\left(t-t_{1}\right) \Delta t \\
& \simeq C_{m}(t) \quad \int_{0}^{t-t_{1}} \int_{0}\left\{\frac{d \tilde{\rho}(x)}{d x}\right)_{x=t-u} a^{2}(t-u)\left(\frac{d a(x)}{d x}\right)_{x=t-u} \\
& +(\Delta t) \\
& \left.+\left(\frac{d^{2} \tilde{\rho}(x)}{d x^{2}}\right)_{x=t-u} a^{3}(t-u)\right\} \theta(u) d u \\
& +(\Delta t)\left(\frac{d \tilde{\rho}(x)}{d x}\right)_{x=t_{1}} a^{3}\left(t_{1}\right) \theta\left(t-t_{1}\right) .
\end{aligned}
$$

Note that

$$
\begin{aligned}
& \int_{0}^{t-t_{1}}\left\{3\left(\frac{d \tilde{\rho}(x)}{d x}\right)_{x=t-u} a^{2}(t-u)\left(\frac{d a(x)}{d x}\right)_{x=t-u}\right. \\
& \left.+\left(\frac{d^{2} \tilde{\rho}(x)}{d x^{2}}\right)_{x=t-u} a^{3}(t-u)\right\} \theta(u) d u \\
& =\int_{0}^{t-t_{1}}\left\{\frac{d}{d x}\left[\left(\frac{d \tilde{\rho}(x)}{d x}\right) a^{3}(x)\right]_{x=t-u}\right\} \theta(u) d u \\
& =\int_{t_{1}}^{t}\left\{\frac{d}{d x}\left[\left(\frac{d \tilde{\rho}(x)}{d x}\right) a^{3}(x)\right]\right\} \theta(t-x) d x \\
& =-\left.\left(\frac{d \tilde{\rho}(x)}{d x}\right) a^{3}(x)\right|_{x=t_{1}} ^{x=t} \theta\left(t-t_{1}\right) .
\end{aligned}
$$

After using (A.25) and (A.26) in (A.18) we find

$$
\dot{C}_{m}(t)=-\left(\frac{d \tilde{\rho}(t)}{d t}\right) a^{3}(t) \theta\left(t-t_{1}\right) \text {. }
$$

In a similar way one may find $\dot{C}_{r}(t)$ as

$$
\begin{aligned}
\dot{C}_{r}(t)= & \int_{0}^{t-t_{1}}\left\{4\left(\frac{d \tilde{\rho}(x)}{d x}\right)_{x=t-u} a^{3}(t-u)\left(\frac{d a(x)}{d x}\right)_{x=t-u}\right. \\
& \left.+\left(\frac{d^{2} \tilde{\rho}(x)}{d x^{2}}\right)_{x=t-u} a^{4}(t-u)\right\} \theta(u) d u \\
& +\left(\frac{d \tilde{\rho}(x)}{d x}\right)_{x=t_{1}} a^{4}\left(t_{1}\right) \theta\left(t-t_{1}\right) \\
= & \left(\frac{d \tilde{\rho}(t)}{d t}\right) a^{4}(t) \theta\left(t-t_{1}\right) .
\end{aligned}
$$

\section{Appendix B. An example for the evolution of the energy den- sities of matter, a resonance with $\omega=-1$, and radiation as a function of scale factor}

In this appendix we derive the evolutions of $C_{m}, \rho_{R}$, and $C_{r}$ and the related quantities in the case

$\omega_{R}=-1, r=7$ and

Initially $s=-\frac{3}{2}$, in between $s=0$,

at late times $s=-2$.

We will try to see what happens in each era explicitly. First we consider the initial times when $t_{1}<t<t_{2}, s=-\frac{3}{2}$ i.e. $H=$ $\xi_{1} a^{-\frac{3}{2}}$ where $\xi_{1}$ is some constant. Then a cosmological constantlike resonance dominated universe where $s=0$ for $t_{2}<t<t_{3}$, Finally, the radiation dominated era at the end where $s=-2$ for $t_{3}<t$. For simplicity We will assume that $\beta$ is constant in all eras. We give $C_{m}, \rho_{R}, C_{r}$ in these eras below by using the formulas derived in the text. $\rho_{m}$ and $\rho_{r}$ are evident from $C_{m}$ and $C_{r}$. For simplicity we assume abrupt changes in the equations of states as one passes from one era to the other although in a more realistic case the changes would be smooth.

$C_{m}$ for $t<t_{2}$ may be directly found from (43) for $r=7$, $s=s_{1}=-\frac{3}{2}, \xi=\xi_{1}$. The result is

$C_{m}=\frac{\rho_{m 0}}{1+\frac{2 \beta \rho_{m 0}}{9 \xi_{1}} \theta\left(t-t_{1}\right)\left(a^{r-s-4}-a_{1}^{r-s-4}\right)}$

where $a=a(t), a_{1}=a\left(t_{1}\right) \cdot \frac{d \tilde{\rho}_{m}(t)}{d t}$ in this case directly follows from Eq. (44)

$$
\begin{aligned}
& \left(\frac{d \tilde{\rho}_{m}(t)}{d t}\right)_{1} \\
& =\frac{81 \theta\left(t-t_{1}\right) \rho_{m 0}^{2} \beta \xi_{1}^{2}}{4\left\{-\frac{9}{2} \xi_{1}+\beta \rho_{m 0} \theta\left(t-t_{1}\right)\left[a^{\frac{9}{2}}\left(t_{1}\right)-a^{\frac{9}{2}}(t)\right]\right\}^{2}} .
\end{aligned}
$$

After using Eq. (21), and Eq. (B.3) we get

$$
\begin{aligned}
C_{R}(t)= & \frac{81}{4} \theta\left(t-t_{1}\right) \rho_{m 0}^{2} \beta \xi_{1}^{-1} \\
& \times \int_{a_{1}}^{a} e^{\Gamma\left(t^{\prime}-t_{1}\right)} \frac{a^{\prime \frac{1}{2}} d a^{\prime}}{\left\{-\frac{9}{2} \xi_{1}+\beta \rho_{m 0}\left[a_{1}^{\frac{9}{2}}-a^{\prime} \frac{9}{2}\right]\right\}^{2}}
\end{aligned}
$$

where $a=a(t), a_{1}=a\left(t_{1}\right)$, and we have used $d t=\frac{d a}{a H}$. One may let $\left|\Gamma\left(t-t_{1}\right)\right| \simeq 0, a_{1}^{\frac{9}{2}}-a^{\frac{9}{2}} \simeq 0$ since we consider the times when the production of $\rho_{R}$ have just started. Hence (B.4) may be approximated by

$C_{R}(t)=\frac{2}{3} \theta\left(t-t_{1}\right) \frac{\rho_{m 0}^{2} \beta}{\xi_{1}}\left[a^{\frac{3}{2}}-a_{1}^{\frac{3}{2}}\right]$

$\rho_{R}(t)$, by (20), becomes

$\rho_{R}(t)=e^{-\Gamma\left(t-t_{1}\right)} \frac{C_{R}}{a^{3}} \simeq \frac{2}{3} \theta\left(t-t_{1}\right) \frac{\rho_{m 0}^{2} \beta}{\xi_{1}} \frac{1}{a^{3}(t)}\left[a^{\frac{3}{2}}-a_{1}^{\frac{3}{2}}\right]$

where $t_{1}<t<t_{2}$.

The corresponding $C_{r}$ is found by using (27) and (B.6) as

$$
\begin{aligned}
C_{r}(t) & =\frac{2 \Gamma \rho_{m 0}^{2} \beta}{\xi_{1}^{2}} \int_{a_{1}}^{a}\left(a^{\prime 3}-a_{1}^{\frac{3}{2}} a^{\prime \frac{3}{2}}\right) d a^{\prime} \\
& =\frac{\Gamma \rho_{m 0}^{2} \beta}{2 \xi_{1}^{2}}\left[a^{4}-\frac{8 a_{1}^{\frac{3}{2}}}{5} a^{\frac{5}{2}}+\frac{3}{5} a_{1}^{4}\right] .
\end{aligned}
$$

At later times we assume the condensate of the resonance dominates, so $s=0$ i.e. $H=\xi_{2}$. To determine $\rho_{R}$ we should first find $C_{m}$ in this case. To find $C_{m}$ in this case we should divide the integral in (42) into two parts; one for the era when $s=-\frac{3}{2}$ between $t_{1}$ and $t_{2}$, and then the era when $s=0$ between $t_{2}$ and $t$. Then the corresponding $C_{m}(t)$ is found as

$$
\begin{aligned}
& -\frac{1}{C_{m}(t)}=-\theta\left(t-t_{1}\right) \beta\left[\frac{1}{\xi_{1}} \int_{a_{1}}^{a_{2}} \frac{d a^{\prime}}{a^{\prime 5-r_{1}+s_{1}}}\right. \\
& +\frac{1}{\xi_{2}} \int_{a_{2}}^{a} \frac{d a^{\prime}}{\left.a^{\prime 5-r_{2}+s_{2}}\right]-\frac{1}{C_{m}\left(t_{1}\right)}} \\
& =\beta \theta\left(t-t_{1}\right)\left\{\frac { 1 } { \xi ( 4 - r _ { 1 } + s _ { 1 } ) } \left[\frac{1}{a^{4-r_{1}+s_{1}\left(t_{2}\right)}}\right.\right.
\end{aligned}
$$




$$
\begin{aligned}
\left(\frac{d \tilde{\rho}_{m}(t)}{d t}\right)_{2} & =-\frac{\dot{C}_{m}(t)}{a^{3}(t)} \\
& =\frac{\theta\left(t-t_{2}\right) \rho_{m 0}^{2} \beta}{\left\{1+\frac{1}{3} \beta \rho_{m 0}\left[\theta\left(t_{2}-t_{1}\right) \frac{2}{3} \xi_{1}^{-1}\left(a_{2}^{\frac{9}{2}}-a_{1}^{\frac{9}{2}}\right)+\theta\left(t-t_{2}\right) \xi_{2}^{-1}\left(a^{3}-a_{2}^{3}\right)\right]\right\}^{2}}
\end{aligned}
$$

Box II.

$$
\begin{aligned}
& \left.\left.-\frac{1}{a^{4-r_{1}+s_{1}\left(t_{1}\right)}}\right]\right\} \\
& +\frac{1}{\xi\left(4-r_{2}+s_{2}\right)}\left[\frac{1}{a^{4-r_{2}+s_{2}(t)}}-\frac{1}{a^{4-r_{2}+s_{2}\left(t_{2}\right)}}\right]-\frac{1}{\rho_{m 0}} \\
& =\left\{\rho _ { m 0 } \beta \left[\theta\left(t_{2}-t_{1}\right) \xi_{2}\left(4-r_{2}+s_{2}\right)\left(a_{1}^{4-r_{1}+s_{1}}-a_{2}^{4-r_{1}+s_{1}}\right)\right.\right. \\
& \times a_{2}^{4-r_{2}+s_{2}} a^{4-r_{2}+s_{2}} \\
& +\theta\left(t-t_{2}\right) \xi_{1}\left(4-r_{1}+s_{1}\right)\left(a_{2}^{4-r_{2}+s_{2}}-a^{4-r_{2}+s_{2}}\right) \\
& \left.\times a_{2}^{4-r_{1}+s_{1}} a_{1}^{4-r_{1}+s_{1}}\right] \\
& -\xi_{1} \xi_{2}\left(4-r_{1}+s_{1}\right)\left(4-r_{2}+s_{2}\right) a_{2}^{4-r_{1}+s_{1}} a_{1}^{4-r_{1}+s_{1}} \\
& \left.\times a_{2}^{4-r_{2}+s_{2}} a^{4-r_{2}+s_{2}}\right\} \\
& \times\left[\rho_{m 0} \xi_{1} \xi_{2}\left(4-r_{1}+s_{1}\right)\left(4-r_{2}+s_{2}\right) a_{2}^{4-r_{1}+s_{1}}\right. \\
& \left.\times a_{1}^{4-r_{1}+s_{1}} a_{2}^{4-r_{2}+s_{2}} a^{4-r_{2}+s_{2}}\right]^{-1} .
\end{aligned}
$$

Hence we find $C_{m}$ in this case as

$$
\begin{aligned}
C_{m}(t)= & \rho_{m 0}\left\{1-\rho_{m 0} \beta\left[\theta\left(t_{2}-t_{1}\right) \xi_{1}^{-1}\left(4-r_{1}+s_{1}\right)^{-1}\right.\right. \\
& \times\left(a_{2}^{r_{1}-4-s_{1}}-a^{r_{1}-4-s_{1}}\right) \\
& +\theta\left(t-t_{2}\right) \xi_{2}^{-1}\left(4-r_{2}+s_{2}\right)^{-1} \\
& \left.\left.\times\left(a^{r_{2}-4-s_{2}}-a_{2}^{r_{2}-4-s_{2}}\right)\right]\right\}^{-1} .
\end{aligned}
$$

Thus, the corresponding $\frac{d \tilde{\rho}_{m}(t)}{d t}$ for $r_{1}=r_{2}=7, s_{1}=-\frac{3}{2}, s_{2}=0$, and $t>t_{2}$ is given in Box II,

where we have skipped the term proportional to the delta function $\delta\left(t-t_{2}\right)$ since it does not contribute to $C_{R}$ below.

$$
\begin{aligned}
C_{R}(t)= & \int_{t_{1}}^{t_{2}} d t^{\prime}\left(\frac{d \tilde{\rho}_{m}\left(t^{\prime}\right)}{d t^{\prime}}\right)_{1} \theta\left(t-t^{\prime}\right) e^{\Gamma\left(t^{\prime}-t_{1}\right)} \\
& +\int_{t_{2}}^{t} d t^{\prime}\left(\frac{d \tilde{\rho}_{m}\left(t^{\prime}\right)}{d t^{\prime}}\right)_{2} \theta\left(t-t^{\prime}\right) e^{\Gamma\left(t^{\prime}-t_{1}\right)} \\
\simeq & \frac{2}{3} \frac{\rho_{m 0}^{2} \beta}{\xi_{1}}\left[a_{2}^{\frac{3}{2}}-a_{1}^{\frac{3}{2}}\right]+\theta\left(t-t_{2}\right) \rho_{m 0}^{2} \beta \xi_{2}^{-1} \\
& \times \int_{a_{2}}^{a} \frac{\left(\frac{a^{\prime}}{a_{1}}\right)^{\gamma} d a^{\prime}}{a^{\prime}\left\{A+B \theta\left(t-t_{2}\right) a^{\prime 3}\right\}^{2}}
\end{aligned}
$$

where $\gamma=\frac{\Gamma}{H}, a(t)=e^{H t}$, and

$A=1+\frac{1}{3} \beta \rho_{m 0}\left[\frac{2}{3} \xi_{1}^{-1}\left(a_{2}^{\frac{9}{2}}-a_{1}^{\frac{9}{2}}\right)-\xi_{2}^{-1} a_{2}^{3}\right]$

$B=\frac{1}{3} \xi_{2}^{-1} \beta \rho_{m 0}$.

In general the result of (B.12) is complicated, so analyzing the result is not easy to see. However one may have an idea on the form of $\rho_{R}$ by analyzing some specific cases. One must have $\Gamma>H$ to insure that the system stays coupled, so the decay may take place. One must also take $\Gamma$ in the same order of magnitude as the Hubble parameter $H$ to insure that the decay process is relevant at cosmological scales. In the light of these observations we may, for example, let $\gamma=3$. Hence (B.12) becomes

$$
\begin{aligned}
C_{R}(t)= & \frac{2}{3} \frac{\rho_{m 0}^{2} \beta}{\xi_{1}}\left[a_{2}^{\frac{3}{2}}-a_{1}^{\frac{3}{2}}\right] \\
& +\theta\left(t-t_{2}\right) \frac{\rho_{m 0}^{2} \beta}{3 B \xi_{2}}\left[\frac{1}{A}-\frac{1}{A+B a^{3}}\right] .
\end{aligned}
$$

It is evident from (B.15) that $C_{R}$ approaches the constant value $\rho_{m 0} a_{1}^{-3}$ and its rate of increase decreases as the scale factor $a$ increases, as expected. $\rho_{R}(t)$, by (20), becomes

$$
\begin{aligned}
& \rho_{R}(t)=\left(\frac{a_{1}}{a}\right)^{3} \frac{1}{3}\left[\frac{2 \rho_{m 0}^{2} \beta}{\xi_{1}}\left\{a_{2}^{\frac{3}{2}}-a_{1}^{\frac{3}{2}}\right\}\right. \\
& \left.+\theta\left(t-t_{2}\right) \frac{\rho_{m 0}^{2} \beta}{B \xi_{2}}\left\{\frac{1}{A}-\frac{1}{A+B a^{3}}\right\}\right]
\end{aligned}
$$

where $t_{2}<t<t_{3}$

here the $\left(\frac{a_{1}}{a}\right)^{3}$ term in the front of (B.16) is due to the $e^{-\Gamma\left(t-t_{1}\right)}$ term in (20). The corresponding $C_{r}$ is found as

$$
\begin{aligned}
C_{r}(t)= & \Gamma\left[\int_{t_{1}}^{t_{2}} \rho_{R}\left(t^{\prime}\right) a^{4}\left(t^{\prime}\right) \theta\left(t-t^{\prime}\right) d t^{\prime}\right. \\
& \left.+\int_{t_{2}}^{t} \rho_{R}\left(t^{\prime}\right) a^{4}\left(t^{\prime}\right) \theta\left(t-t^{\prime}\right) d t^{\prime}\right] \\
= & \frac{\Gamma \rho_{m 0}^{2} \beta}{2 \xi_{1}^{2}}\left[a_{2}^{4}-\frac{8 a_{1}^{\frac{3}{2}}}{5} a_{2}^{\frac{5}{2}}+\frac{3}{5} a_{1}^{4}\right] \\
& +\frac{\Gamma \rho_{m 0}^{2} \beta a_{1}^{3}}{3 \xi_{2}} \int_{a_{2}}^{a}\left[\frac{2}{\xi_{1}}\left\{a_{2}^{\frac{3}{2}}-a_{1}^{\frac{3}{2}}\right\}\right. \\
& \left.+\frac{1}{B \xi_{2}}\left\{\frac{1}{A}-\frac{1}{A+B a^{\prime 3}}\right\}\right] d a^{\prime} \\
= & \frac{\Gamma \rho_{m 0}^{2} \beta}{2 \xi_{1}^{2}}\left[a_{2}^{4}-\frac{8 a_{1}^{\frac{3}{2}}}{5} a_{2}^{\frac{5}{2}}+\frac{3}{5} a_{1}^{4}\right] \\
& +\frac{2 \Gamma \rho_{m 0}^{2} \beta a_{1}^{3}}{3 \xi_{1} \xi_{2}}\left(a_{2}^{\frac{3}{2}}-a_{1}^{\frac{3}{2}}\right)\left(a-a_{2}\right) \\
& +\frac{\Gamma \rho_{m 0}^{2} \beta a_{1}^{3}}{3 B \xi_{2}^{2}}\left[\frac{a-a_{2}}{A}+\left(6 A^{\frac{2}{3}} B^{\frac{1}{3}}\right)^{-1}\right. \\
& {[}
\end{aligned}
$$




$$
\begin{aligned}
& \times\left\{2 \sqrt{3} \tan ^{-1}\left(\frac{1-\frac{2 B^{\frac{1}{3}}}{A^{\frac{1}{3}}} a}{\sqrt{3}}\right)\right. \\
& -2 \sqrt{3} \tan ^{-1}\left(\frac{1-\frac{2 B^{\frac{1}{3}}}{A^{\frac{1}{3}}} a_{2}}{\sqrt{3}}\right) \\
& -2 \ln \left(\frac{A^{\frac{1}{3}}+B^{\frac{1}{3}} a}{A^{\frac{1}{3}}+B^{\frac{1}{3}} a_{2}}\right) \\
& \left.+\ln \left(\frac{A^{\frac{2}{3}}-A^{\frac{1}{3}} B^{\frac{1}{3}} a+B^{\frac{2}{3}} a^{2}}{A^{\frac{2}{3}}-A^{\frac{1}{3}} B^{\frac{1}{3}} a_{2}+B^{\frac{2}{3}} a_{2}^{2}}\right)\right\} .
\end{aligned}
$$

The rate of change $C_{r}$ in (B.17), and if it increases for reasonable values of parameters is not evident from (B.17) since the form of the expression is rather complicated. Therefore one may check the rate of change of $C_{r}$ in (B.17) for a few values of phenomenologically viable sets of parameters. The results of the corresponding $C_{r}$ versus $a$ plots suggest that $C_{r}$ increases as $\propto a$ with the scale factor in this era, $t_{1}<t<t_{2}$. For example, for $\rho_{m 0} \simeq \rho_{c}=$ $9 \times 10^{-30} \mathrm{~g} \mathrm{~cm}^{-3}, \beta^{\prime}=\frac{1}{4}, \sigma\left(t_{1}\right)=10^{-27} \mathrm{~cm}^{2}, v\left(t_{1}\right)=10^{5} \mathrm{~cm} / \mathrm{s}$, $n_{0}=4 \times 10^{-5} \mathrm{~cm}^{-3}$ (where $\beta^{\prime}, \sigma\left(t_{1}\right), v\left(t_{1}\right)$ are defined in (30) and $n_{0}$ is the number density of matter particles at time $t_{1}$ ) gives $\xi_{1}=\sqrt{\frac{8 \pi G}{3} \rho_{m 0}} \simeq 2.2 \times 10^{-3} \mathrm{~s}^{-1}, \beta \rho_{m 0}=10^{-27} \mathrm{~s}^{-1}$. Moreover one may relate $\xi_{2}$ to $\xi_{1}$ by $\xi_{1}=a_{1}^{\frac{3}{2}} \xi_{2}$ where we have used the fact that at time $t_{1}$ the total energy density in a unit comoving volume $a^{3}$ lost by the matter i.e. $\rho_{m 0}=\frac{3 \xi_{1}^{2}}{8 \pi G}$ is equal to the one obtained by the resonance i.e. to $\frac{3 \xi_{3}^{2} a^{3}}{8 \pi G}$. $\xi_{1}=a_{1}^{\frac{3}{2}} \xi_{2}$ implies that $\xi_{2}>2.2 \times 10^{-3} \mathrm{~s}^{-1}$. Therefore $A \simeq 1, B \simeq 0$, and from the definition of $B$ we have $\xi_{2} B=\frac{1}{3} \beta \rho_{m 0}$. In this approximation the last two lines in (B.17) cancel after expanding $\tan ^{-1}$ and $\ln$ by Taylor expansion and keeping the leading order terms and then letting $B=0, A=1$. Thus (B.17) may be approximated by

$$
\begin{aligned}
C_{r}(t) \simeq & \frac{\Gamma \rho_{m 0}}{\xi_{1}}\left[\frac{\rho_{m 0} \beta}{2 \xi_{1}}\left(a_{2}^{4}-\frac{8 a_{1}^{\frac{3}{2}}}{5} a_{2}^{\frac{5}{2}}+\frac{3}{5} a_{1}^{4}\right)\right. \\
& \left.+\left(\frac{2 \rho_{m 0} \beta a_{1}^{3}\left(a_{2}^{\frac{3}{2}}-a_{1}^{\frac{3}{2}}\right)}{3 \xi_{2}}\right)\left(a-a_{2}\right)\right] .
\end{aligned}
$$

We see that $C_{r}$ continues to increase in the era, $t_{1}<t<t_{2}$ while its rate of increase gets smaller as expected.

Finally we consider the last era of the conversion of matter to radiation through formation of a resonance i.e. the radiation dominated era after conversion of most of matter to radiation i.e. when $s=-2$. We find $C_{m}^{-1}$ in this case in a similar way as done in (B.9), that is,

$$
\begin{aligned}
\frac{1}{C_{m}(t)}= & \frac{\beta}{\xi_{1}} \int_{a_{1}}^{a_{2}} \frac{d a^{\prime}}{a^{\prime 5-r_{1}+s_{1}}}+\frac{\beta}{\xi_{2}} \int_{a_{2}}^{a_{3}} \frac{d a^{\prime}}{a^{\prime 5-r_{2}+s_{2}}}+\frac{1}{C_{m}\left(t_{1}\right)} \\
& +\frac{\theta\left(t-t_{3}\right) \beta}{\xi_{3}} \int_{a_{3}}^{a} \frac{d a^{\prime}}{a^{\prime 5-r_{1}+s_{1}}} .
\end{aligned}
$$

Then $C_{m}$ for $t>t_{3}$ is found as

$$
C_{m}(t)=\frac{\rho_{m 0}}{A^{\prime}+\theta\left(t-t_{3}\right) B^{\prime} a^{5}}
$$

where $A^{\prime}, B^{\prime}$ are some constants that, for $r_{1}=r_{2}=r_{3}=7, s_{1}=-\frac{3}{2}$, $s_{2}=0, s_{1}=-2$, given by

$$
\begin{aligned}
A^{\prime}= & 1+\frac{1}{3} \beta \rho_{m 0}\left[\frac{2}{3} \xi_{1}^{-1}\left(a_{2}^{\frac{9}{2}}-a_{2}^{\frac{9}{2}}\right)+\xi_{2}^{-1}\left(a_{3}^{3}-a_{2}^{3}\right)\right] \\
& -\frac{a_{3}^{5} \theta\left(t-t_{3}\right)}{5 \xi_{3}}, B^{\prime}=\frac{1}{5} \xi_{3}^{-1} \beta \rho_{m 0} .
\end{aligned}
$$

The corresponding $\frac{d \tilde{\rho}_{m}(t)}{d t}$ is

$$
\left(\frac{d \tilde{\rho}_{m}(t)}{d t}\right)_{3}=-\frac{\dot{C}_{m}(t)}{a^{3}(t)}=\theta\left(t-t_{3}\right) \frac{\rho_{m 0} B H a^{2}}{\left(A+B a^{5}\right)^{2}}
$$

where we have skipped the term proportional to the delta function $\delta\left(t-t_{2}\right)$ since it does not contribute to $C_{R}$ below. Thus $C_{R}$ is found as

$$
\begin{aligned}
C_{R}(t)= & \frac{2}{3} \frac{\rho_{m 0}^{2} \beta}{\xi_{1}}\left[a_{2}^{\frac{3}{2}}-a_{1}^{\frac{3}{2}}\right] \\
& +\theta\left(t-t_{2}\right) \frac{1}{3 B \xi_{2}} \rho_{m 0}^{2} \beta\left[\frac{1}{A}-\frac{1}{A+B a_{3}^{3}}\right] \\
& +B \rho_{m 0} \theta\left(t-t_{3}\right) \int_{a_{3}}^{a} e^{\frac{\Gamma}{2 \xi_{3}}\left(a^{\prime 2}-a_{1}^{2}\right)} \frac{a^{\prime} d a^{\prime}}{\left\{A^{\prime}+B^{\prime} a^{\prime 5}\right\}^{2}}
\end{aligned}
$$

where we have used the fact that $H=\xi_{3} a^{-2}$ implies $t-t_{1}=$ $\frac{1}{2 \xi_{3}}\left(a^{2}-a_{1}^{2}\right)$. The integral in (B.23) cannot be evaluated exactly even with use of Mathematica. However one may give an approximate result as follows. At the final stages of the resonance we have $\Gamma\left(t-t_{1}\right) \sim 1$, so $x=1-\Gamma\left(t-t_{1}\right) \ll 1$. Therefore

$e^{\Gamma\left(t-t_{1}\right)}=e^{1-x}=e e^{-x} \simeq e(1-x)=e \Gamma\left(t-t_{1}\right)$.

Hence the integral in (B.23) may be approximated as

$$
\begin{aligned}
& \int_{a_{3}}^{a} e^{\frac{\Gamma}{2 \xi_{3}}\left(a^{\prime 2}-a_{1}^{2}\right)} \frac{a^{\prime} d a^{\prime}}{\left\{A^{\prime}+B^{\prime} a^{\prime 5}\right\}^{2}} \\
& \simeq \int_{a_{3}}^{a} \frac{e \Gamma}{2 \xi_{3}} \frac{\left(a^{\prime 2}-a_{1}^{2}\right) a^{\prime} d a^{\prime}}{\left\{A^{\prime}+B^{\prime} a^{\prime}\right\}^{2}} \simeq \frac{e \Gamma}{2 \xi_{3}} \int_{a_{3}}^{a} \frac{a^{\prime 3} d a^{\prime}}{\left\{A^{\prime}+B^{\prime} a^{\prime}\right\}^{2}} .
\end{aligned}
$$

The result of (B.25) is rather complicated so that it makes it difficult to get concrete results from the expression. However one may draw its plots for several values of $A^{\prime}$ and $B^{\prime}$. The indefinite form of (B.25) for all tried values of $A^{\prime}, B^{\prime}$ give flat graphs for almost all values of $a(t)$. For example, for reasonable values of parameters discussed after Eq. (B.17) and using the conservation of energy in a comoving volume at time $t_{2}$ i.e. $\xi_{2} a_{2}^{\frac{3}{2}}=\xi_{3} a_{2}^{-\frac{1}{2}}$ i.e. $\xi_{3}=$ $\xi_{2} a_{2}^{2}=\xi_{1} a_{1}^{-\frac{3}{2}} a_{2}^{2}$ if the resonance lives a sufficiently long time so that $a_{2} \gg a_{1}$ then we have $\xi_{3}<\xi_{1}<\xi_{2}$. Otherwise $\xi_{1}$ and $\xi_{3}$ must be comparable. Therefore $A^{\prime} \simeq 1, B^{\prime} \simeq 0$. We have given the result of this integration for two three different ratios of $A^{\prime}$ and $B^{\prime}$ in the Figs. 2-4. Therefore the result of (B.25) is almost zero for all phenomenologically viable cases. In other words in this final era i.e. for $t>t_{3} C_{R}$ does not increase any more and $\rho_{R}$ decays exponentially

$$
\begin{aligned}
\rho_{R}(t)= & e^{\Gamma\left(t-t_{1}\right)} C_{R}(t) \\
\simeq & \frac{4 \xi_{3} \rho_{m 0}^{2} \beta}{3 e \Gamma \xi_{1}}\left(a^{2}-a_{1}^{2}\right)^{-1} \\
& \times\left[a_{2}^{\frac{3}{2}}-a_{1}^{\frac{3}{2}}+\theta\left(t-t_{2}\right) \frac{a_{1}^{\frac{3}{2}}}{2 B}\left(1-\frac{1}{1+B a_{3}^{3}}\right)\right] .
\end{aligned}
$$




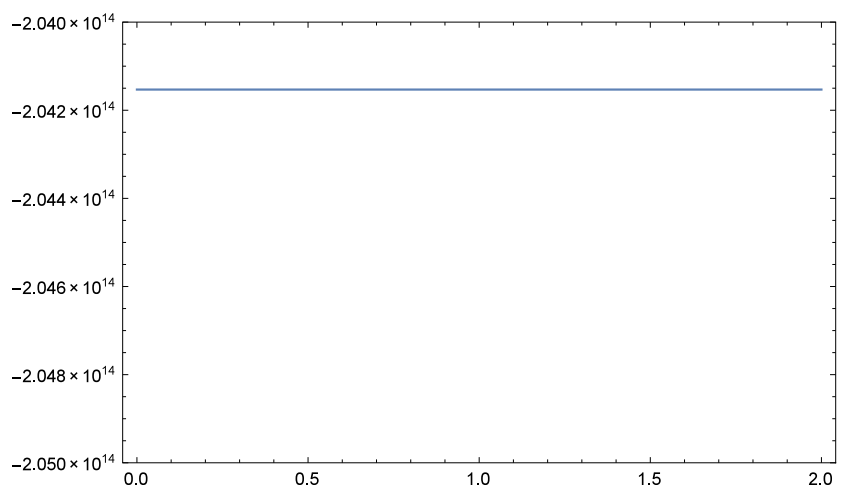

Fig. 2. The value of the integral in Eq. (B.25) as a function of scale factor for $A^{\prime}=1$, $B^{\prime}=10^{-20}$.

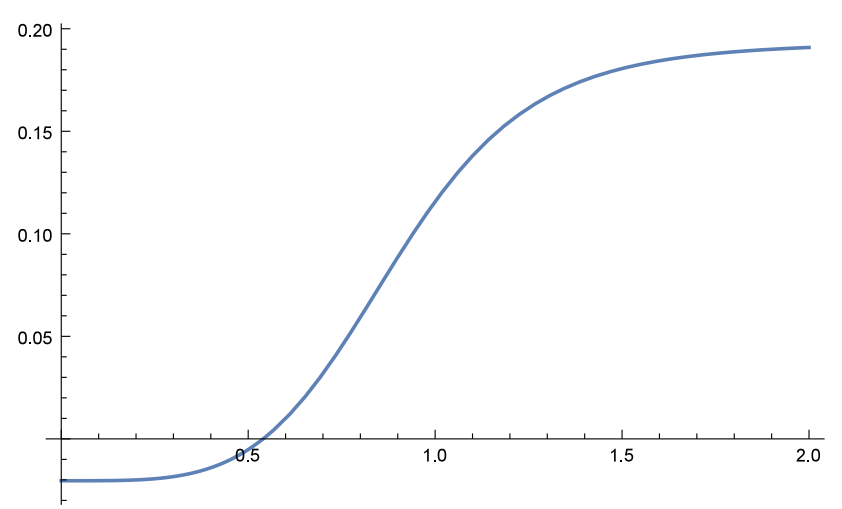

Fig. 3. The value of the integral in Eq. (B.25) as a function of scale factor for $A^{\prime}=1$, $B^{\prime}=1$.

Then the corresponding $C_{r}$ becomes

$$
\begin{aligned}
C_{r}(t) \simeq & \frac{\Gamma \rho_{m 0}}{\xi_{1}}\left[\frac{\rho_{m 0} \beta}{2 \xi_{1}}\left(a_{2}^{4}-\frac{8 a_{1}^{\frac{3}{2}}}{5} a_{2}^{\frac{5}{2}}+\frac{3}{5} a_{1}^{4}\right)\right. \\
& \left.+\left(\frac{2 \rho_{m 0} \beta a_{1}^{3}\left(a_{2}^{\frac{3}{2}}-a_{1}^{\frac{3}{2}}\right)}{3 \xi_{2}}+a_{1}^{\frac{9}{2}}\right)\left(a_{3}-a_{2}\right)\right] \\
& +C^{\prime} \int_{a_{3}}^{a} \frac{a^{\prime 5} d a^{\prime}}{a^{\prime 2}-a_{1}^{2}}
\end{aligned}
$$

where

$C^{\prime}=\frac{2 \rho_{m 0}^{2} \beta}{3 e \xi_{1}}\left[2\left(a_{2}^{\frac{3}{2}}-a_{1}^{\frac{3}{2}}\right)+\frac{a_{1}^{\frac{3}{2}}}{B}\left(1-\frac{1}{1+B a_{3}^{3}}\right)\right]$

for $t>t_{3}$.

After integration of the integral in (B.27) we obtain

$$
\begin{aligned}
C_{r}(t) & \simeq \frac{\Gamma \rho_{m 0}}{\xi_{1}}\left[\frac{\rho_{m 0} \beta}{2 \xi_{1}}\left(a_{2}^{4}-\frac{8 a_{1}^{\frac{3}{2}}}{5} a_{2}^{\frac{5}{2}}+\frac{3}{5} a_{1}^{4}\right)\right. \\
& \left.+\left(\frac{2 \rho_{m 0} \beta a_{1}^{3}\left(a_{2}^{\frac{3}{2}}-a_{1}^{\frac{3}{2}}\right)}{3 \xi_{2}}+a_{1}^{\frac{9}{2}}\right)\left(a_{3}-a_{2}\right)\right] \\
& +C^{\prime}\left[\frac{a_{1}^{2}}{2}\left(a^{2}-a_{3}^{2}\right)+\frac{a^{4}-a_{3}^{4}}{4}+\frac{a_{1}^{4}}{2} \ln \left(\frac{a^{2}-a_{1}^{2}}{a_{3}^{2}-a_{1}^{2}}\right)\right] .
\end{aligned}
$$

We notice that there is a term proportional to $a^{4}$. Although the coefficient of this much smaller than the others it is anomalous

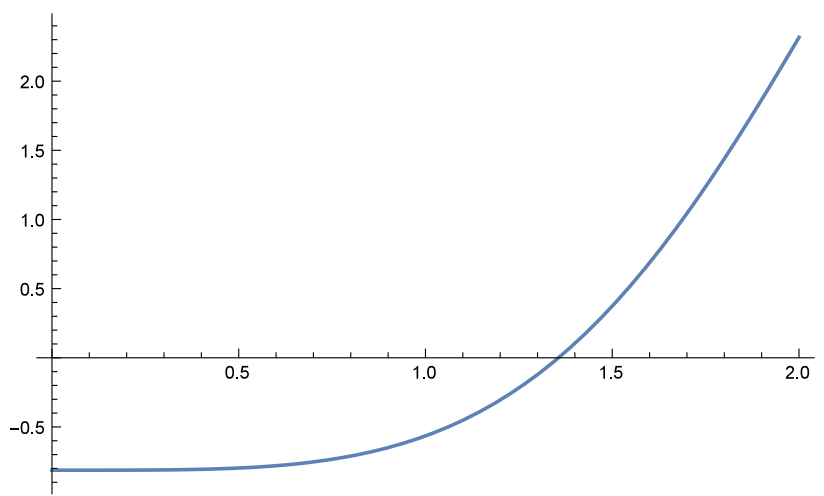

Fig. 4. The value of the integral in Eq. (B.25) as a function of scale factor for $A^{\prime}=1$, $B^{\prime}=0.01$.

since it makes the production of radiation seem gets faster while it should be go to a constant value at the end of the decay. In fact this anomalous behavior is due to identifying the time when $\Gamma\left(t-t_{1}\right) \simeq 1$ with radiation dominated era. Taking this time as a radiation dominated era is an incorrect identification since about one third of resonance particles with $s=0$ survive at this time and the increase in $C_{r}$ due to decay of resonance makes it effectively behave as an energy density with equation of state smaller than $\frac{1}{3}$. Therefore taking $s=-2$ is not valid unless a small amount of resonance particles survive and hence the rate of conversion of resonance particles to radiation becomes very small. Therefore one must take $s=-2$ at a much later time. For example one may take $\Gamma\left(t-t_{1}\right) \simeq 4$ i.e.

$$
\begin{aligned}
& \frac{\Gamma\left(t-t_{1}\right)}{4} \sim 1 \Rightarrow x=1-\frac{\Gamma\left(t-t_{1}\right)}{4} \ll 1 \\
& \Rightarrow e^{\Gamma\left(t-t_{1}\right)}=e^{4(1-x)}=e^{4}\left(e^{-x}\right)^{4} \\
& \simeq e^{4}(1-x)^{4}=e^{4}\left(\frac{\Gamma\left(t-t_{1}\right)}{4}\right)^{4}=\left(\frac{e \Gamma}{8 \xi_{3}}\right)^{4}\left(a^{2}-a_{1}^{2}\right)^{4} .
\end{aligned}
$$

After substituting (B.30) in the first term in (B.25) and using Mathematica to evaluate the integral and draw the corresponding plots we get almost zero for the result of the integration as before for reasonable values of parameters mentioned before. Next we use (B.30) to find $\rho_{R}$. Then (B.26) is replaced by

$$
\begin{aligned}
\rho_{R}(t)= & e^{\Gamma\left(t-t_{1}\right)} C_{R}(t) \simeq \frac{4096 \xi_{3}^{4} \rho_{m 0}^{2} \beta}{3 e^{4} \Gamma^{4} \xi_{1}}\left(a^{2}-a_{1}^{2}\right)^{-4} \\
& \times\left[2\left(a_{2}^{\frac{3}{2}}-a_{1}^{\frac{3}{2}}\right)+\theta\left(t-t_{2}\right)\right. \\
& \left.\times \frac{a_{1}^{\frac{3}{2}}}{B}\left(1-\frac{1}{1+B a_{3}^{3}}\right)\right] \text { for } t>t_{3} .
\end{aligned}
$$

Then the corresponding $C_{r}$ becomes

$$
\begin{aligned}
C_{r}(t) \simeq & \frac{\Gamma \rho_{m 0}}{\xi_{1}}\left[\frac{\rho_{m 0} \beta}{2 \xi_{1}}\left(a_{2}^{4}-\frac{8 a_{1}^{\frac{3}{2}}}{5} a_{2}^{\frac{5}{2}}+\frac{3}{5} a_{1}^{4}\right)\right. \\
& \left.+\left(\frac{2 \rho_{m 0} \beta a_{1}^{3}\left(a_{2}^{\frac{3}{2}}-a_{1}^{\frac{3}{2}}\right)}{3 \xi_{2}}+a_{1}^{\frac{9}{2}}\right)\left(a_{3}-a_{2}\right)\right] \\
& +C^{\prime \prime} \int_{a_{3}}^{a} \frac{a^{\prime 5} d a^{\prime}}{\left(a^{\prime 2}-a_{1}^{2}\right)^{4}}
\end{aligned}
$$


where

$$
\begin{aligned}
C^{\prime \prime}= & \frac{4096 \xi_{3}^{3} \rho_{m 0}^{2} \beta}{3 e^{4} \Gamma^{3} \xi_{1}}\left[2\left(a_{2}^{\frac{3}{2}}-a_{1}^{\frac{3}{2}}\right)+\frac{a_{1}^{\frac{3}{2}}}{B}\right. \\
& \left.\times\left(1-\frac{1}{1+B a_{3}^{3}}\right)\right] \text { for } t>t_{3} .
\end{aligned}
$$

The result of the integral in (B.32) is

$$
\begin{aligned}
\int_{a_{3}}^{a} \frac{a^{\prime 5} d a^{\prime}}{\left(a^{\prime 2}-a_{1}^{2}\right)^{4}}= & \frac{a_{1}^{2}-3 a_{1}^{2} a^{2}+3 a^{4}}{6\left(a^{2}-a_{1}^{2}\right)} \\
& -\frac{a_{1}^{2}-3 a_{1}^{2} a_{3}^{2}+3 a_{3}^{4}}{6\left(a_{3}^{2}-a_{1}^{2}\right)}
\end{aligned}
$$

which goes to a constant value (i.e. $C_{r}$ goes to a constant value) as $a$ increases as expected. One may check that later times (that may be taken as the time that satisfy $\Gamma\left(t-t_{1}\right) \simeq k$ where $\left.k>4\right) C_{r}$ goes to the constant value even faster.

\section{References}

[1] S. Weinberg, Cosmology, Oxford Univ. Press, New York, 2008.

[2] L. Amendola, S. Tsujikawa, Dark Energy: Theory and Observations, Cambridge Univ. Press, UK, 2015

[3] E. Gunzig, J. Géhéniau, I. Prigogine, Entropy and cosmology, Nature 330 (1987) 621.

[4] J.A.S. Lima, I. Baranov, Gravitationally induced particle production: Thermodynamics and kinetic theory, Phys. Rev. D 90 (2014) 043515 arXiv:1411.6589, and the references there in.

[5] R. Erdem, A way to get rid of cosmological constant and zero point energy problems of quantum field theory through metric reversal symmetry, J. Phys. A 41 (2008) 235401 arXiv:0712.2989; and the references there in.

[6] S. Pan, S. Bhattacharya, S. Chakraborty, An analytic model for interacting dark energy and its observational constraints, Mon. Not. R. Astron. Soc. 452 (2015) 3038. arXiv: 1210.0396.

[7] R. Erdem, Single scale factor for the universe from the creation of radiation and matter till the Present, Eur. Phys. J. C 74 (2014) 3066. arXiv:1407.7199.

[8] K. Enqvist, D.G. Figueroa, N. Lerner, Curvaton decay by resonant production of the Standard Model higgs, J. Cosmol. Astropart. Phys. 01 (2013) 0240. arXiv: 1211.5028 .

[9] C. Patrignani, et al. (Particle data group), Review of particle Physics, Chin. Phys. C 40 (2016) 100001

[10] V. Faraoni, J.B. Dent, E.N. Sarikadis, Covariantizing the interaction between dark energy and dark matter, Phys. Rev. D 90 (2014) 063510. arXiv:1405.7288; N. Tamanini, Phenomenological models of dark energy interacting with dark matter, Phys. Rev. D 92 (2014) 043524 arXiv:1504.07397; and the references there in.

[11] P. Langacker, The Standard Model and beyond, CRC Press, USA, 2010.

[12] V. Kuksa, Complex-mass definition and the structure of the unstable particle's propagator, Adv. High Energy Phys. 2015 (2015) 490238.
[13] M. Srednicki, Quantum Field Theory, Cambridge University Press, UK, 2007; partially hp-th/0409035. Lecture notes of Modern Quantum Field Theory course given by Einan Gardi in the University of Edinburgh; www2.ph.ed.ac. uk/ egardiMQFTMQFT_2015_lecture_9_10.pdf.

[14] P.T. Matthews, A. Salam, Relativistic Theory of Unstable Particles II, Phys. Rev. 115 (1959) 1079.

[15] A.D. Dolgov, F.R. Urban, Evaporation of charged bosonic condensate in cosmology, Astropart. Phys. 24 (2005) 289.

[16] L.A. Urena-Lopez, Bose-Einstein condensation of relativistic scalar field dark matter, J. Cosmol. Astropart. Phys. 0901 (2001) 014. arXiv:0806.3093.

[17] M.P. Silverman, R.L. Mallet, Cosmic degenerate matter: a possible solution to the problem of missing mass, Gen. Relativity Gravitation 18 (2001) L37; M.P. Silverman, R.L. Mallet, Dark matter as a cosmic Bose-Einstein condensate and possible superfuid, Gen. Relativity Gravitation 34 (2002) 633;

T. Harko, P. Liang, S.-D. Liang, G. Mocanu, Testing the Bose-Einstein Condensate dark matter model at galactic cluster scale, J. Cosmol. Astropart. Phys, 1511 (2015) 027, arXiv:1510.06275. and the references therein.

[18] M. Nishiyama, M. Morita, M. Morikawa, Bose-Einstein condensation as dark energy and dark matter, preprint, astro-ph/0403571.

[19] T. Fukuyama, M. Morikawa, T. Tatekawa, Cosmic structures via bose-Einstein condensation and its collapse, J. Cosmol. Astropart. Phys. 0806 (2008) 033. arXiv:0705.3091.

[20] J. Besprosvany, G. Izquierdo, Coincidence problem with dark energy as a coupled self-interacting Bose-Einstein gas, Classical Quantum Gravity 32 (2015) 055015, arXiv:1405.2296;

S. Das, R. Bhaduri, Dark matter and dark energy from a Bose-Einstein condensate, Classical Quantum Gravity 32 (2015) 105003. arXiv:1411.0753.

[21] T. Fukuyama, M. Morikawa, Stagflation: Bose-Einstein condensation in the early universe, Phys. Rev. D 80 (2009) 063520. arXiv:0905.0173.

[22] S. Das, bose-Einstein condensation as an alternative to Inflation, Internat. J. Modern Phys. D 24 (2015) 1544001. arXiv:1509.02658.

[23] C.J. Pethick, H. Smith, Bose-Einstein Condensation in Dilute Gases, Cambridge Univ. Press, UK, 2002.

[24] L. Pitaevskii, S. Stringari, Bose-Einstein Condensation, Oxford Univ. Press, New York, 2003.

[25] T. Paul, K. Richter, P. Schlagheck, Non-linear resonant transport of BoseEinstein condensates, Phys. Rev. Lett. 94 (2005) 020404;

E. Kengne, R. Vaillancourt, Exact solutions of the Gross-Pitaevskii in periodic potential in the presence of external source, J. Math. Phys. 48 (2007) 073520; T.S. Raju, P.K. Panigrahi, Exact solutions of the modified Gross-Pitaevskii equation in 'smart' periodic potentials in the presence of external source, J. Nonlinear Math. Phys. 18 (2011) 367.

[26] H.E. Haber, H.A. Weldon, Finite-temperature symmetry breaking as boseEinstein condensation, Phys. Rev. D 25 (1982) 502.

[27] H.T.C. Stoof, Formation of the condensate in a dilute bose gas, Phys. Rev. Lett. 66 (1991) 3148; $\quad$ H.T.C. Stoof, Nucleation of Bose-Einstein condensation, Phys. Rev. A 45 (1992) 8398.

[28] C.W. Gardiner, M.D. Lee, R.J. Ballagh, M.J. Davis, P. Zoller, Quantum kinetic theory of condensate growth: Comparison of experiment theory, Phys. Rev. Lett. 81 (1998) 5266.

[29] A.S. Parkins, D.F. Walls, The physics of trapped dilute gas bose-Einstein condensates, Phys. Rep. 303 (1998) 1.

[30] E. Di Valentino, S. Gariazzo, M. Gerbino, E. Giusarma, Dark radiation inflationary freedom after Planck 2015, Phys. Rev. D 93 (2016) 083523:

M. Archidiacona, E. Giusarma, S. Hannestad, O. Mena, Cosmic dark radiation and neutrinos, Adv. High Energy Phys. 2013 (2013) 191047.

[31] R.J. Scherrer, M.S. Turner, Decaying particles do not "heat up" the Universe, Phys. Rev. D 31 (1985) 681 